\title{
Mindfulness meditation alters neural activity underpinning working memory during tactile distraction
}

\author{
Michael Yufeng Wang ${ }^{1,2} \cdot$ Gabrielle Freedman $^{1} \cdot{\text { Kavya } \text { Raj }^{3} \cdot \text { Bernadette Mary Fitzgibbon }^{1,2} \cdot \text { Caley Sullivan }}^{2} \cdot$ \\ Wei-Lin $\operatorname{Tan}^{1} \cdot$ Nicholas Van Dam ${ }^{4,5}$ - Paul B Fitzgerald ${ }^{1,2} \cdot$ Neil W Bailey $^{2}$
}

Accepted: 29 August 2020 / Published online: 24 September 2020

(C) The Psychonomic Society, Inc. 2020

\begin{abstract}
Evidence suggests that mindfulness meditation (MM) improves selective attention and reduces distractibility by enhancing topdown neural modulation. Altered P300 and alpha neural activity from MM have been identified and may reflect the neural changes that underpin these improvements. Given the proposed role of alpha activity in supressing processing of task-irrelevant information, it is theorised that altered alpha activity may underlie increased availability of neural resources in meditators. The present study investigated attentional function in meditators using a cross-modal study design, examining the P300 during working memory (WM) and alpha activity during concurrent distracting tactile stimuli. Thirty-three meditators and 27 healthy controls participated in the study. Meditators showed a more frontal distribution of P300 neural activity following WM stimuli $(p$ $\left.=0.005, \eta^{2}=0.060\right)$ and more modulation of alpha activity at parietal-occipital regions between single (tactile stimulation only) and dual task demands (tactile stimulation plus WM task) $\left(p<0.001, \eta^{2}=0.065\right)$. Additionally, meditators performed more accurately than controls $\left(p=0.038, \eta^{2}=0.067\right)$. The altered distribution of neural activity concurrent with improved WM performance suggests greater attentional resources dedicated to task related functions, such as WM in meditators. Thus, meditation-related neural changes are likely multifaceted, involving both altered distribution and also amplitudes of brain activity, thereby enhancing attentional processes depending on task requirements.
\end{abstract}

Keywords Attention $\cdot$ Working memory $\cdot$ Cognitive control $\cdot$ Mindfulness $\cdot$ Electroencephalography

It is theorised that the effects of mindfulness meditation (MM) on cognitive performance are mediated by improved neural

Electronic supplementary material The online version of this article (https://doi.org/10.3758/s13415-020-00828-y) contains supplementary material, which is available to authorized users.

Neil W Bailey

neil.bailey@monash.edu

1 Monash Alfred Psychiatry Research Centre, Monash University Central Clinical School, Commercial Rd, Melbourne, Victoria, Australia

2 Epworth Centre for Innovation in Mental Health, the Epworth Clinic, 888 Toorak Road, Camberwell, Victoria, Australia

3 Brain and Mental Health Research Hub, School of Psychological Sciences, Monash Institute of Cognitive and Clinical Neurosciences, and Monash Biomedical Imaging, Monash University,

Clayton, VIC 3168, Australia

4 School of Psychological Sciences, The University of Melbourne, Parkville, VIC, Australia

5 Department of Psychiatry, Icahn School of Medicine at Mount Sinai, New York, NY, USA resource allocation during stimuli processing, thereby prompting rapid attention allocation and reallocation speed, enhancing cognitive efficiency (Malinowski, 2013; Moore, Gruber, Derose, \& Malinowski, 2012; van Leeuwen, Singer, \& Melloni, 2012). Enhanced attentional processing, awareness, and sustained focus are considered to be core mechanistic components of many mindfulness-based practices (Lippelt, Hommel, \& Colzato, 2014; Lutz, Slagter, Dunne, \& Davidson, 2008), and MM has been demonstrated to improve selective attention and reduce distractibility (Moore et al., 2012; Wong, Teng, Chee, Doshi, \& Lim, 2018; Zeidan, Johnson, Diamond, David, \& Goolkasian, 2010b). These changes result in enhanced performance of resource demanding cognitive processes, evidence of which has been shown in tasks that require executive functions, memory, and selfregulation (Chiesa, Calati, \& Serretti, 2011; Manna et al., 2010; Tang \& Posner, 2009). For example, meditatorsboth novice and experienced - outperformed controls on a generalized attention task, demonstrating greater attentional functioning (Jha, Krompinger, \& Baime, 2007). These improvements may be related to altered neural activity (Fox 
et al., 2016) and structure (Fox et al., 2014) in meditators and provide insights into the underlying neural basis of attentional changes through MM.

An important neural marker of mindfulness related performance enhancements has been found in electroencephalography (EEG) studies using event-related-potentials (ERPs) exploring task-dependent P300 modulation (Slagter et al., 2007). The P300 is a positive voltage deflection typically peaking around $300 \mathrm{~ms}$ after presentation of a target stimulus. It is likely related to activity in the frontal and temporal-parietal networks and functions to facilitate selective attention and visual working memory ([WM]; Polich, 2007). The P300 is considered an index of resource allocation during complex task performances, and its amplitudes have shown correlations with increased demand for evaluative resources (Kok, 2001; Polich, 2007; Slagter et al., 2007). Meditators have shown reductions in attentional-blink effects (where the second stimulus in a sequence of rapidly presented stimuli is commonly missed; Slagter et al., 2007), along with reductions in P300 amplitudes to the first stimuli (Slagter et al., 2007). Mindfulness practice has also been associated with reduced interference from distractor stimuli and greater control of resource allocation across multiple modalities (auditory and visual; van den Hurk, Giommi, Gielen, Speckens, \& Barendregt, 2010), more efficient neural processing and attentional network functioning (Isbel, Lagopoulos, Hermens, \& Summers, 2019), and improved ability to direct attentional resources toward task relevant stimuli (Moore et al., 2012).

Although evidence suggests mindfulness practice can influence neural resource allocation, the mechanism by which this occurs is unclear. One theory proposes that mindfulness related attention enhancements are the result of practicespecific effects on alpha modulation (Kerr, Sacchet, Lazar, Moore, \& Jones, 2013). Modulation of the alpha rhythm has been observed to regulate sensory inputs to the somatosensory cortex and is seen as a filtering mechanism in a range of information processing tasks (Foxe \& Snyder, 2011). For example, alpha modulation has been found to correlate with WM load and performance, with greater alpha power over sensory processing areas during higher memory load, and greater alpha power associated with higher probabilities of correct WM responses (Jensen, Bonnefond, \& VanRullen, 2012; Jensen \& Mazaheri, 2010; Scheeringa et al., 2009). Some research has suggested that increases in alpha activity function to allow internally allocated attentional processes (of which WM retention is a subset; Jensen et al., 2012; Klimesch, 2012). In support of this point, greater magnitudes of alpha activity have been found during periods of selfreported mind wandering compared to periods of behavioural task performance (Baldwin et al., 2017; Compton, Gearinger, \& Wild, 2019). Mind-wandering is a process where attention is directed internally toward a stream of thought rather than externally toward a task or environmental stimuli (Compton et al., 2019). Conversely, reductions in alpha activity has been found during stimuli presentation and sensory stimulation (Thut, Nietzel, Brandt, \& Pascual-Leone, 2006). This suggests that greater alpha activity may represent greater internally directed attentional processes while lower alpha activity may reflect externally directed processes.

In meditators, modulation of alpha in the primary somatosensory cortex appears to facilitate sensory throughput when people are cued to direct attention to specific body regions (Jones et al., 2010). Thus, modulation of alpha over sensory regions appears to act as a suppression mechanism during resource demanding cognitive processes by reducing distractor processing (Sauseng et al., 2009). Together, mindfulness-meditation likely improves cognitive performance during resource demanding internally focused tasks (such as WM) through greater alpha activity in brain regions related to external stimuli processing (such as sensory regions), supressing irrelevant stimuli processing (Kerr et al., 2013).

At the present, preliminary evidence suggests that mindfulness related cognitive enhancements may be enabled by 1) increased attention to the target stimuli (with increases in associated neural activity), 2) increased suppression of taskirrelevant sensory information, potentially via higher alpha activity over task-irrelevant cortical regions, or 3) a combination of both. However, previous methods have not permitted dissociation of these possibilities. A better understanding of the mechanism by which mindfulness exerts its effects could facilitate a better understanding of when and for what conditions it might be helpful.

The current study used a cross-modal design combining a WM task with a tactile distractor to allow for specific testing of the role of target-enhancing and distractor-suppressing mechanisms in attentional processing. The study investigated differential processing of concurrently presented visual and somatosensory stimuli in meditators and controls by examining P300 ERPs related to visual WM stimuli and alpha activity related to tactile stimuli. In particular, we tested whether meditators would demonstrate enhanced WM related electrophysiological responses concurrent with greater ability to suppress sensory processing (reflected by higher alpha activity over somatosensory regions). We predicted that meditators would show enhanced ERP amplitudes to WM stimuli, reflecting enhanced attention despite distraction, irrespective of whether distractors required a response. Secondly, research has indicated that meditators demonstrate more pronounced frontal ERPs when attending to task-relevant stimuli, reflecting more engagement of attention-relevant areas (N. Bailey et al., 2018). As such, we expected WM-related ERPs in the current study to show more frontal distributions among meditators (again irrespective of whether distractors required a response). Third, preliminary evidence has indicated enhanced ability in meditators to modulate alpha activity in regions processing 
distractor stimuli (Kerr et al., 2013). With the current study design, this effect was tested in two ways. First, when only tactile stimuli were presented (no visual stimuli), increased alpha activity was expected in visual processing regions in meditators, reflecting suppression of non-relevant visual processing regions. Second, when WM stimuli were presented concurrent with a tactile distractor, increased alpha activity in somatosensory processing regions was expected in meditators, reflecting suppression of nonrelevant somatosensory information.

Our primary hypotheses were as follows:

1) Meditators were expected to show larger $P 300$ amplitudes toward visual WM stimuli than the control group, indicating increased processing of WM stimuli.

2) Meditators were expected to show a more frontal distribution of the P300 to WM stimuli reflecting increased engagement of attentional processing regions.

3) Meditators were expected to show greater alpha activity over somatosensory regions (time locked to tactile stimuli) during the visual WM task than controls.

4) Meditators were expected to show greater alpha activity over visual processing regions during task conditions requiring tactile-only processing, reflecting suppression of task irrelevant brain regions for additional neural resources.

Furthermore, a post-hoc exploratory analysis was performed to determine whether meditators showed more modulation of somatosensory alpha depending on whether tactile stimuli were attended to or not in replication of Kerr et al. (2013). It was hypothesised that results would overlap with Kerr et al. (2013), with meditators showing greater power in alpha activity when ignoring tactile stimulation compared with controls. Exploratory source analyses were also planned to characterise the source of neural activation differences between groups (without statistical comparisons) and exploratory microstate analyses were planned to characterise the shifting pattern of neural activity across the period after WM stimuli presentation. Lastly, as neural data was the focus of this study, behavioural comparisons were exploratory without specific directional hypotheses.

\section{Methods}

\section{Participants}

Seventy participants between the ages of 18 to 65 ( 45 females and 25 males) were recruited for the study: 34 mindfulness meditators and 36 healthy control nonmeditators. Participants were recruited via community, meditation centres, and university advertising and were reimbursed a total $\$ 30$ for their participation.

Meditation participants were included if they had practiced for more than 2 years and currently practiced more than 2 hours per week. Screening was conducted through phone and in-person interview by experienced mindfulness researchers $(\mathrm{GF}, \mathrm{KR}, \mathrm{NWB})$ to ensure mindfulness practice were congruent with Kabat-Zinn's definition"paying attention in a particular way: on purpose, in the present moment, and nonjudgmentally" (Pregi, Vittori, Pérez, Leirós, \& Nesse, 2006). Screening also ensured meditation practices were consistent with either focused attention on the breath or body scan. Uncertainties were resolved through discussion between the principle researcher (NWB) and a second researcher. Control group participants were excluded if they reported more than 2 hours of lifetime meditation experience.

Exclusion criteria included self-reported current or past experiences of mental or neurological illness, current psychoactive medication, or recreational drug use. These were further assessed by using the Mini International Neuropsychiatric Interview for DSM-IV (Sheehan et al., 1998), Beck Depression Inventory-II (BDI-II), and Beck Anxiety Inventory (BAI) (Beck \& Clark, 1997; Beck, Steer, \& Brown, 1996), which were administered by GF, KR, or NWB. Participants were excluded if they met diagnostic criteria for any DSM-IV psychiatric disorders or if they scored in the mild or above range on the Beck anxiety and depression scales.

Prior to completing the task, participants provided demographic information and reported their estimated years of mindfulness practice and minutes per week of current practice. Self-report measures were also completed; Freiburg Mindfulness Inventory (FMI; Walach, Buchheld, Buttenmüller, Kleinknecht, \& Schmidt, 2006), Five Facet Mindfulness Questionnaire (FFMQ; Baer, Smith, Hopkins, Krietemeyer, \& Toney, 2006), BAI, and BDI. Written informed consent was obtained from participants prior to the commencement of the study. The Monash University Human Research Ethics Committee and the Alfred Hospital Ethics Committee approved all experimental procedures.

Four participants in the control group were excluded due to high scores on the BDI (within clinical range) and one control was excluded due to task noncompletion. To maximise data available for analysis, further exclusions of select data were made for neural analysis separately. Four controls and one meditator were excluded from the neural analysis, as they provided too few artefact free EEG epochs for analysis (based on the criteria provided in the Electrophysiological Recording and Data Processing section). Thus, final analyses were run on 27 controls and 33 meditators for neural analysis and 31 controls and 34 meditators for behavioural analysis. 


\section{Procedure}

To study the effect of cross-modal task demands on attention and related neural activity in meditators, we used a two group (meditators vs. controls) $\times$ two condition (attend/ignore tactile stimulation) design across two task conditions with different sensory modalities (visual working memory/tactile oddball), with four within subject conditions in total (Fig. 1). Each participant was tested in one continuous session split in four different testing conditions while EEG was recorded (details of the EEG recording below). In the first condition, participants experienced brief tactile sensations (comprising a tactile oddball), which they were instructed to ignore (Condition [C] 1: Ignore Tactile Only). In the second condition, participants continued to experience and were instructed to ignore the tactile sensations, while concurrently performing an N-back WM task (2-back), pressing button 1 for target letters (Condition 2: Ignore Tactile, Attend N-back). In the third condition, participants experienced tactile sensations without the N-back task being present, and were instructed to respond by pressing button 2 after the infrequent occurrence of two sensations presented in close temporal proximity (Condition 3: Attend Tactile Only). The final condition involved participants responding with one button to the N-back task, and with another button to the infrequently presented double tactile stimuli (Condition 4: Attend Tactile, Attend N-back).

In the first testing condition, participants were administered 125 brief electrotactile stimuli and instructed to remain quietly seated while ignoring the stimuli by directing their gaze toward the screen in front without any particular attentional focus (condition 1: Ignore Tactile Only). Participants were

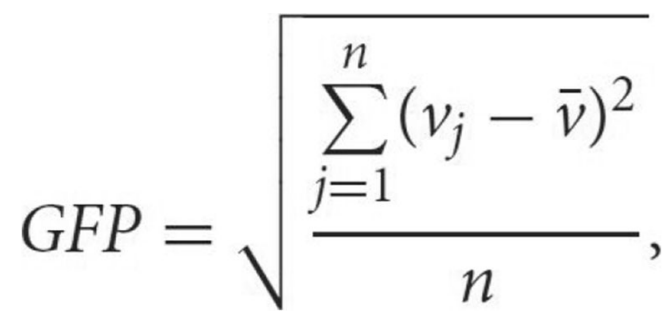

Fig. 2 Equation for calculation of the Global Field Potential as outlined by Habermann et al. (2018). Note: $\mathrm{j}$ is the channel index, $\mathrm{vj}$ is the voltage measured at channel $\mathrm{j},-\mathrm{v}$ is the mean voltage value across all channels (i.e., the average reference) and $\mathrm{n}$ is the number of channels

not required to make a response in this condition. The electrotactile stimuli were administered to the anterior radial portion of the left wrist (median nerve) using a transducer with two flat metal probes (cathode proximal) of 9-mm diameter at 30-mm spacing apart (Stimulating Bar Electrode, MLADDF30, AD Instruments, NSW, Australia). Electrical current was passed between these two probes for $80 \mathrm{~ms}$ during each pulse and was delivered by digitally generated waveforms converted to analogue signal and then amplified (Powerlab, AD Instruments, FE116, NSW, Australia). The amplitude of electrical stimulation was set at $1.75 \mathrm{x}$ the sensory threshold (which was determined by delivering randomly timed brief electrical stimulations at amplitudes slowly increasing from 0.5 volts until participants consistently reported detecting the sensation, then reducing the amplitude until participants could no longer detect the sensation). The sensory threshold was defined as the amplitude 0.1 volts above the value at which participants reported detecting the sensation with $100 \%$ accuracy out of five electrotactile stimulations.

\begin{tabular}{|c|c|c|c|c|}
\hline & $\begin{array}{l}\text { Condition 1: } \\
\text { Ignore Tactile } \\
\text { Only }\end{array}$ & $\begin{array}{l}\text { Condition 2: } \\
\text { Ignore Tactile, } \\
\text { Attend N-back }\end{array}$ & $\begin{array}{l}\text { Condition 3: } \\
\text { Attend Tactile } \\
\text { Only }\end{array}$ & $\begin{array}{l}\text { Condition 4: } \\
\text { Attend Tactile, } \\
\text { Attend N-back }\end{array}$ \\
\hline Task & $\begin{array}{l}\text { Tactile } \\
\begin{array}{lll} & & \\
\cdot & \cdot\end{array}\end{array}$ & 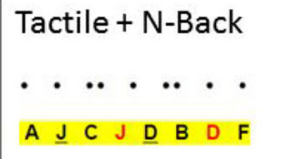 & $\begin{array}{l}\text { Tactile } \\
\text {. . • .. }\end{array}$ & 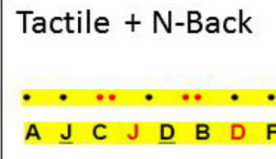 \\
\hline Attention Load & None & $\begin{array}{l}\text { Attention to visual } \\
\text { stimuli }\end{array}$ & $\begin{array}{l}\text { Attention to } \\
\text { tactile stimuli }\end{array}$ & $\begin{array}{l}\text { Attention to visua } \\
\text { and tractile } \\
\text { stimuli }\end{array}$ \\
\hline $\begin{array}{l}\text { Neural Markers } \\
\text { Investigated }\end{array}$ & $\begin{array}{l}\text { Tactile related } \\
\text { alpha activity }\end{array}$ & $\begin{array}{l}\text { WM related ERP } \\
\text { and tactile related } \\
\text { alpha activity }\end{array}$ & $\begin{array}{l}\text { Tactile related } \\
\text { alpha activity }\end{array}$ & $\begin{array}{l}\text { WM related ERP } \\
\text { and tactile related } \\
\text { alpha activity }\end{array}$ \\
\hline
\end{tabular}

Fig. 1 Task design. All participants completed conditions 1-4 in the same order. Dots represent the tactile stimulation; single dots represent a single tactile stimulation, double dots represent a target oddball double tactile stimulation, red double dots highlight the target during attend conditions (requiring response with button 2). Letters represent the N-back WM letters; underlined letters represent the first letter in the target pair, red letters represent memory targets to which a response is required (with button 1). Yellow represents the direction of attention according the task instructions 
Amplitude was set at $1.75 \mathrm{x}$ the sensory threshold stimulation value to account for variation in participants' sensory threshold. Participants confirmed the sensation as noticeable but not eliciting discomfort, and all participants included in analyses provided responses to the tactile stimuli in conditions where they were required to do so, confirming that the sensations were detected. Amplitude of the electrical signal varied between 0.7 and 2.3 volts depending on each individual's threshold. Oddball stimuli consisted of double stimulations (with an inter-stimulus interval of $250 \mathrm{~ms}$ ) and were randomly dispersed throughout the series accounting for $16 \%$ of the trials. The same stimulation was used to deliver both single standard stimulations (random inter-trial interval of 1,800-2,840 ms, with an average of 2,320 ms) and oddball stimulations.

During the second condition, the N-back WM task was introduced, and participants were asked to attend to the Nback task while continuing to ignore the tactile stimulation (the 125 regular and oddball tactile stimulations were presented through the second condition; Condition 2: Ignore Tactile, Attend N-back). The N-back memory task was presented on a computer approximately $80 \mathrm{~cm}$ in front of participants in a darkened and sound attenuated room. A series of letters (from A to J) were presented in random order. Participants responded by pressing button 1 when the currently viewed letter was the same as the letter presented two trials previously (2-back). A short practice task was provided before initiation of the real task. The complete N-back task consisted of 390 visual WM stimuli, in two blocks of 195 , with a 1.5 second inter-trial interval and trials contained $25 \%$ of target letters. The second condition was designed to provide a measure of neural activity related to WM during ongoing tactile distraction (described below). The timing of tactile stimulations had no relationship to the presentation of memory stimuli in all blocks and was identical to the sequence in the Ignore Tactile Only condition in all conditions.

The third condition presented the tactile oddball, but not the N-back task. Participants were required to attend to the 125 tactile stimuli and press button 2 when they felt the oddball double stimulation (Condition 3: Attend Tactile Only). The fourth condition presented both the 125 tactile stimuli and the N-back stimuli concurrently. Participants were asked to continue attending to the tactile stimuli pressing 2 for an oddball double stimulation, and at the same time perform the N-back task, pressing button 1 for a target letter (Condition 4: Attend Tactile, Attend $\mathrm{N}$-back). In this fourth condition, participants were asked to split their attention between dual tasks, allowing measurement of WM ERPs and alpha activity to tactile stimulation during an attention-limited state with greater task demands. Task transitions were demarcated by new instructions informing participants of changes in the task requirements.

\section{Data Analysis}

\section{Behavioural Data Comparisons}

Tactile oddball and N-back response accuracies were compared by first calculating d-prime scores $\left(d^{\prime}=z\right.$ [hit rate]- $z$ [false alarm rate]), because this measure allows for more accurate comparison of differences in behavioural performance (Wickens, 2010). $d$ ' scores were then analysed for each type of stimuli separately by using a repeated measures ANOVA in SPSS, as were reaction times to both types of stimuli separately. $d$ ' was compared by using a repeated-measures ANOVA for response to $\mathrm{N}$-back letters ( 2 groups $\mathrm{x} 2$ conditions $-\mathrm{C} 2$ : Ignore Tactile, Attend N-back/C4: Attend Tactile, Attend Nback) and for response to tactile stimulation (2 groups $\mathrm{x} 2$ conditions - Attend C3: Tactile Only/C 4: Attend Tactile, Attend N-back). Reaction time was compared by using a repeated-measures ANOVA for response to N-back letters ( 2 groups $\times 2$ conditions - C2: Ignore Tactile, Attend Nback/C4: Attend Tactile, Attend N-back) and for response to tactile stimulation (2 groups $\mathrm{x} 2$ conditions $-\mathrm{C} 1$ : Attend Tactile Only/C4: Attend Tactile, Attend N-back). As recommended by Tabachnick and Fidell (2013), a value larger than 3.29SD from the mean was used to detect outliers. Based on this criterion, no outliers were detected in the present study. There was no evidence for violation of univariate or multivariate equality of variance nor for violations of normality.

\section{Electrophysiological Recording and Data Processing}

A 64-channel Neuroscan EEG Ag/AgCl Quick Cap acquired data to Neuroscan software through a SynAmps2 amplifier (Compumedics, Melbourne, Australia). Electrodes were referenced online to an electrode between $\mathrm{Cz}$ and $\mathrm{CPz}$. Horizontal and vertical eye movements were recorded by using four EOG electrodes located above and below the left orbit and adjacent to the outer canthus of each eye. Impedances were maintained at less than $5 \mathrm{k} \Omega$. Recordings were sampled at $1,000 \mathrm{~Hz}$ and bandpass filtered from 0.05 to $200 \mathrm{~Hz}$ ( $24 \mathrm{~dB} /$ octave roll off). MATLAB (The Mathworks, Natick, MA, 2016a) and EEGLAB were used for pre-processing of EEG data (sccn.ucsd.edu/eeglab; Delorme \& Makeig, 2004). Independent component analysis (ICA) was used to remove artefacts (described in more detail shortly). The performance of ICA is adversely affected when data below $1 \mathrm{~Hz}$ is included (Anemüller, Sejnowski, \& Makeig, 2003; Delorme \& Makeig, 2004), so second-order Butterworth filtering was applied to the data with a bandpass from $1-80 \mathrm{~Hz}$ and a band stop filter 47-53 Hz. Data were then epoch time-locked to the onset of the single pulse tactile stimulation $(-1,000$ to $2,000 \mathrm{~ms})$ and also epoch time-locked to the onset of the visual stimuli in the WM conditions ( $-1,000$ to $2,000 \mathrm{~ms})$. 
All analyses of alpha activity were performed on data time-locked to single pulse tactile stimuli rather than to oddball stimuli. The task design presented single-pulse stimuli at greater frequency than the oddball stimulus. This provided more neural data and allowed more reliable interpretations. Participants were not instructed to respond to the single-pulse tactile stimuli, and epochs containing responses to single-pulse tactile stimuli or to the N-back stimuli were excluded to avoid confounding motor activity. Epochs time-locked to the onset of visual WM stimuli also excluded epochs with responses. Epochs were visually inspected by an experimenter experienced with EEG analysis and periods containing muscle artefact or excessive noise were excluded as were channels with low quality signals. Each participant provided 35 or more accepted epochs for each condition - the number of accepted epochs between conditions was not significant (all $p$ values $>0.10$ ). To avoid excluding low-frequency brain activity, which can adversely affect ERP comparisons, such as P300s that contain activity below $1 \mathrm{~Hz}$ (Tanner, Morgan-Short, \& Luck, 2015), and to ensure independent component analysis was performed effectively (Anemüller et al., 2003; Delorme \& Makeig, 2004), adaptive mixture independent component analysis was used to first manually select and remove components related to eye movement and remaining muscle (Palmer, Makeig, Kreutz-Delgado, \& Rao, 2008). Raw data were then refiltered from 0.1 to $80 \mathrm{~Hz}$ with artifact rejections from all previous channels, epochs, and the ICA step applied to this data (Palmer et al., 2008). This ensured that both the ICA and the ERP analysis steps were optimal as recommended by previous research (Anemüller et al., 2003; Bailey et al., 2019; Delorme \& Makeig, 2004). The refiltered data was again inspected by a separate researcher blinded to the group identity of data inspected at the time. Recordings were re-referenced offline to an averaged reference. Epochs were then averaged within each WM condition and each participant for statistical analysis of ERPs. Alpha activity related to tactile stimuli was computed using a Morlet Wavelet transform with a 3.5-cycle width to provide a measure of power in the $8-$ to $13-\mathrm{Hz}$ alpha frequency range. These power values were averaged across epochs for each of the conditions separately for each participant.

\section{Statistical Comparisons}

Self-report and behavioural results were analysed by using SPSS version 23. Independent samples $t$-tests were performed to examine potential group differences in age, years of education, BDI, and BAI, FMI, and FFMQ scores. Potential differences in categorical data (gender and handedness) were examined by using the Chi-square test.

\section{Primary Comparisons}

Statistical comparisons of EEG data were conducted using the Randomization Graphical User Interface (RAGU), which uses rank-order randomization statistics to compare scalp field differences from all electrodes and epoch timepoints between groups (Koenig, Kottlow, Stein, \& Melie-García, 2011). RAGU computes the Global Field Power (GFP) for each time point across the epoch to represent the strength of neural activity across all channels. Using the GFP as a single value in the statistical analysis controls for multiple comparisons across the spatial dimension (Habermann, Weusmann, Stein, \& Koenig, 2018). GFP is a reference-free EEG measure, which avoids an arbitrary choice of reference (Koenig et al., 2011). The GFP calculation is equivalent to the standard deviation across all channels, and the equation as outlined by Habermann et al. (2018) is:

Global duration statistics were used to control for multiple comparisons across time for the ERP data (alpha comparisons did not require multiple comparison control in the temporal dimension as alpha activity was averaged across the 0 to $1,000 \mathrm{~ms}$ window after stimuli presentation). Global duration statistics calculated the duration of significant effects within the epoch that are longer than $95 \%$ of significant periods in the randomized data. In addition, Global count statistics were used to ensure the risk of false positive significant results was not increased, by testing whether the count of significant timepoints across the total epoch in the real data exceeded the count of significant timepoints in $95 \%$ of the randomised data (global count control) (Habermann et al., 2018).

Ragu also allows for the comparison of scalp distributions of neural activity with the Topographic Analysis of Variance (TANOVA). The recommended L2 normalization was performed to normalize differences in individual neural response amplitude, so that TANOVA comparisons test differences in the distribution of neural activity independently of differences in amplitude. To assess consistent topographical activation, the voltage value from each electrode at each timepoint within each individual is averaged within each condition and group. The mean-GFP is computed from this averaged map. Consistent activation from each individual within a condition and group will result in a larger mean-GFP than inconsistent activation. Then, to create a null distribution for the TCT test, the voltage value from each electrode is randomly shuffled within each individual before being averaged across all individuals to create a null distribution of mean-GFPs. This approach preserves the GFP value for each individual, but results in a small mean-GFP as any consistency across individuals in topography of voltage reflecting neural activity in response to the stimulus is destroyed by the randomisation process (Habermann et al., 2018). Comparing the actual data to this null distribution indicates whether the data shows a mean- 
GFP signal that is larger than $95 \%$ of the null distribution or shows consistency that is significant at $p<0.05$.

For the GFP and TANOVA tests, we were interested in between group comparisons rather than consistency within a group (as per the TCT test). As such, shuffling for these tests is implemented by randomly swapping group and condition labels so that in each random permutation, a certain number of meditators will be arbitrarily labelled as controls and vice versa (and the same is applied for each condition). This creates a null distribution where any potential effect of interest are eliminated. Comparing the real data to this distribution gives an indication of whether the real data shows a signal that is larger than $95 \%$ of the null distribution, or significant at $\mathrm{p}<$ 0.05 (Habermann et al., 2018).

Comparisons of ERP data related to WM stimuli were made for the entire 0 to $1000 \mathrm{~ms}$ window following the stimuli. GFP and TANOVA tests were used to conduct 2 group $\times 2$ condition (C2: Ignore Tactile, Attend N-back/C: Attend Tactile, Attend N-back) comparisons for WM ERPs. Comparisons of alpha power were made using averaged activity over the 0 to $1000 \mathrm{~ms}$ window following the onset of the tactile stimulus. Regarding alpha comparisons, it should be noted that when frequency transformed data comparisons are performed with RAGU, the average reference is not computed with the transformed data (the average reference was computed prior to the transforms). As such, the test is a comparison of the Root Mean Square (RMS) between groups. The RMS analysis compares the strength of EEG signals in the frequency domain. RMS is computed in the same manner as the GFP, with the only difference being that RMS values are not rereferenced to the global average first, as frequency transformed data is comprised only of positive values (in contrast to absolute voltages, which show positive voltages in one direction and negative voltages in the other when generated by an underlying dipole in the brain; Ruggeri, Meziane, Koenig, \& Brandner, 2019). In other respects, the statistic used to compare RMS between groups is identical to the GFP test described in the previous paragraph. Alpha values were compared with RMS and TANOVA tests (to separately compare overall neural response strength and distribution of neural activity, respectively). RMS and TANOVA tests were used to conduct 2 group x 2 condition (C2: Ignore Tactile, Attend N-back/C4: Attend Tactile, Attend N-back) x 2 condition (C1: Ignore Tactile Only/C3: Attend Tactile Only) comparison for tactile related alpha activity.

The recommended 5,000 randomization runs were employed for each statistical test. Global duration statistics were used to control for multiple comparisons across time for the ERP data (alpha comparisons did not require multiple comparison control in the temporal dimension as alpha activity was averaged across the 0 to 1,000 -ms window after stimuli presentation). Global duration statistics calculated the duration of significant effects within the epoch that are longer than $95 \%$ of significant periods in the randomized data. This ensures that significant differences in the real data last longer than the random comparison data with our alpha level of 0.05 (Grieder et al., 2012). Refer to Koenig and Melie-García (2010) and Koenig et al. (2011) for further information regarding these analyses.

The Benjamini and Hochberg false discovery rate (FDR; Benjamini \& Hochberg, 1995) was performed on the global count $p$-values from each main effect or interaction. The FDR reduces the false discovery rate and is used to control multiple comparisons for all comparisons involving the primary hypothesis separately from behavioural comparisons. $P$ values are labelled "FDR $p$ " and " $p$-uncorrected" to allow for comparison with other research.

\section{Exploratory Analysis}

To determine whether previous results showing increased modulation of alpha in the meditation group depending on whether stimuli were attended or ignored (Kerr et al., 2013), RMS and TANOVA tests were used to conduct 2 group $\mathrm{x} 2$ condition (C1: Ignore Tactile Only/C3: Attend Tactile Only) comparison for tactile related alpha activity. Source analysis and microstates analysis were used to further explore differences in ERPs following visual stimuli. Microstates are transient patterns of scalp topography lasting from milliseconds to seconds before transitioning to another temporarily stable topography and are hypothesised to be the basic building blocks of neural functioning (Koenig et al., 2002). Ragu was used to identify and time periods that showed significant differences in the between group ERP comparisons. Results of the source and microstate analysis are reported in the Supplementary Materials.

Following a reviewer's suggestion, Bayes Factor analyses (with analogous statistical designs to the frequentist analyses) were used to calculate the probability of the null hypothesis (BF01) or alternative hypothesis (BF10) (Rouder, Morey, Verhagen, Swagman, \& Wagenmakers, 2017). For analyses involving more than a single factor, comparisons were made between models containing a hypothesized effect to equivalent models stripped of the effect. Note that comparisons using Bayesian analyses are not currently possible for the distribution of neural activity, which compare the topographical difference map to a null distribution of topographical difference maps, rather than using unique individual values that enable comparisons of group means. To perform the Bayes Factor analysis on the GFP values, a window of interest between $300 \mathrm{~ms}$ and $600 \mathrm{~ms}$ for the P300 was selected based on previous literature (Linden, 2005; Polich, 2007; Walhovd \& Fjell, 2003). It should be noted that this window was not included in our a priori hypotheses, so results of this comparison must be considered exploratory. 


\section{Results}

\section{Demographics}

Neural analyses were the focus of this study, so only participants selected for neural analyses were examined for differences in demographic and self-report data. Results are summarised in Table 1. No significant differences were found between groups in age, BAI and BDI scores, gender or handedness (all $p>0.3$, all BF01 $>3$ except for BDI, for which BF01 = 2.556). However, meditators scored significantly higher on the FMI, $t(58)=2.401, p=0.019$, and FFMQ, $t(58)=3.741, p<0.001$, compared with controls. Meditators also exhibited more years of education $t(58)=2.01, p=0.049$, $\mathrm{BF} 10=1.404$. Because the difference between groups could be a confound, we replicated all significant comparisons after excluding the six meditators with the highest number of years of education. After exclusion of those six meditators, groups did not differ in years of education $(t(52)=0.925, p=0.359$, BF01 = 2.543). All significant results remained significant when groups without differences in years of education were compared $(p<0.05$, with larger effect sizes found in all tests, reported in Supplementary Materials).

\section{Behavioural Performance}

For N-back results, a significant main effect of group was found for $d$ ', with meditators performing more accurately than controls $\left(\mathrm{F}(1,63)=4.516, p=0.038, \eta^{2}=0.067\right)$. No significant main effect was found for reaction time nor for the interaction between Group $\mathrm{x}$ visual WM conditions (C2: Ignore Tactile, Attend N-back/C4: Attend Tactile, Attend N-back conditions for reaction time or d' (all $p>0.5$, BF01 for the combined model $=8.232$ ). Additionally, for responses to the tactile stimulation, no main effect of group nor interaction between Group x Tactile conditions (C1: Attend Tactile
Only/C4: Attend Tactile, Attend N-back conditions) were found in d' or reaction time (all $p>0.07, \mathrm{BF} 01$ for the combined model $=5.186$ ). Figure 3 presents $d$ ' scores and reaction times in behavioural performances. For a complete table of means, standard deviations, and statistics for behavioural performance comparisons, please see Supplementary Table 1.

\section{Neural Data; Visual Stimuli Locked ERPs}

\section{TCT and GFP}

The TCT was conducted to assess the consistency of neural activity within each group and condition (Koenig et al., 2011), and global count statistics were used to ensure the risk of false positive significant results was not increased (Habermann et al., 2018). The TCT showed topographical consistency within all groups and conditions indicating that TANOVA comparisons are valid during the majority of time periods (see supplementary materials 1 for a more detailed description). The GFP randomization test was performed to assess the strength of ERP neural response to N-back stimuli in C2: Ignore Tactile, Attend N-back and C4: Attend Tactile, Attend N-back conditions. There was no significant main effect of Group (global count statistics across the whole epoch $p$ $=0.280$, FDR $p=0.350$ ), nor interaction for Group $\mathrm{x} \mathrm{C} 2$ : Ignore Tactile, Attend N-back/C4: Attend Tactile, Attend Nback conditions in GFP (no periods of significance lasted longer than the duration control of $47 \mathrm{~ms}$, global count statistics across the whole epoch $p=0.198$, FDR $p=0.282$ ). See Fig. 4.

\section{TANOVA}

TANOVAs were conducted to examine neural activity distribution in response to the $\mathrm{N}$-back stimuli during the visual WM conditions (C2: Ignore Tactile, Attend N-back/C4: Attend

Table 1. Demographics and self-report data

\begin{tabular}{|c|c|c|c|}
\hline $\mathrm{N}$ & $\begin{array}{l}\text { Meditators } M(S D) \\
33\end{array}$ & $\begin{array}{l}\text { Control } M(S D) \\
27\end{array}$ & Statistics \\
\hline $\operatorname{Sex}(\mathrm{F} / \mathrm{M})$ & $21 / 12$ & $17 / 10$ & $X^{2}(1)=0.0029, p=0.957$ \\
\hline Age & $36.91(10.85)$ & $35.07(13.87)$ & $t(58)=0.575, p=0.568$ \\
\hline Years of education & $17.09(2.49)$ & $15.79(2.45)$ & $t(58)=2.011, p=0.049^{*}$ \\
\hline Meditation experience (years) & $8.43(10.41)$ & 0 & \\
\hline Current meditation practice per week (hours) & $5.24(3.93)$ & 0 & \\
\hline BDI score & $1.09(1.89)$ & $1.67(2.69)$ & $t(58)=0.971, p=0.336$ \\
\hline BAI score & $4.33(4.72)$ & $4.78(5.55)$ & $t(58)=0.335, p=0.739$ \\
\hline FMI score & $45.75(7.08)$ & $40.93(8.06)$ & $t(58)=2.470, p=0.016^{*}$ \\
\hline FFMQ score & $153.58(17.02)$ & $138.11(12.83)$ & $t(58)=3.899, p<0.001 * *$ \\
\hline
\end{tabular}

$* p<0.05, * * p<0.001$ 

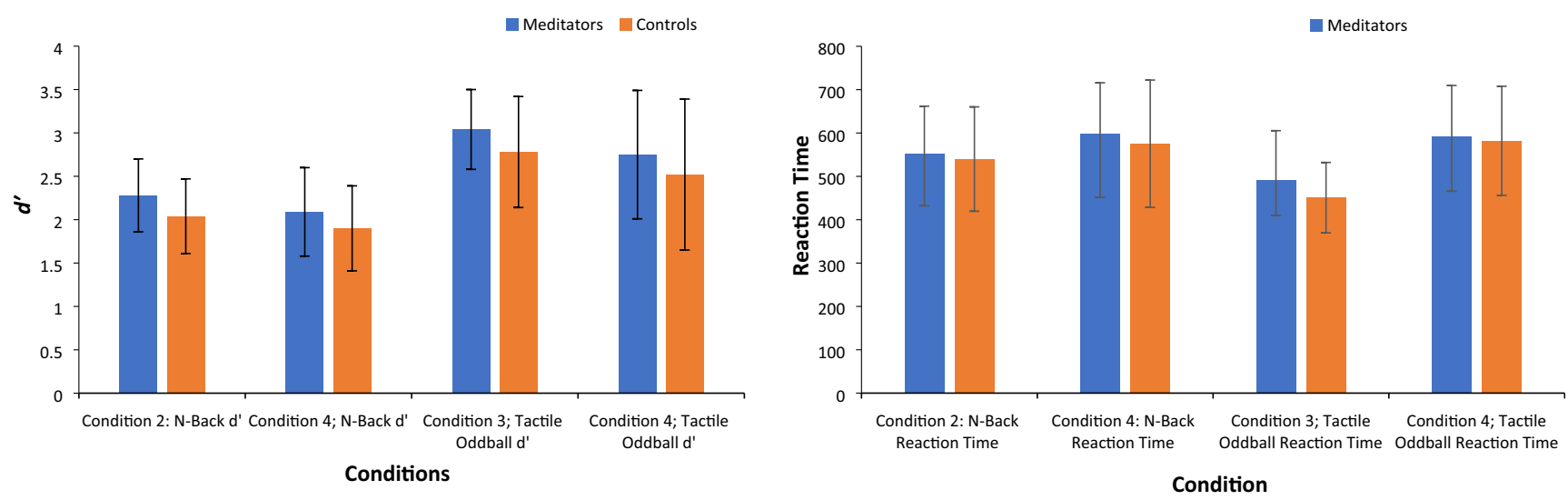

Fig. 3 Signal detection $\left(d^{\prime}\right)$ scores and reaction times in behavioural performance. A. Mean N-Back and tactile oddball $d$ ' scores. Error bars represent standard deviation. B. Mean N-back and tactile oddball reaction times. Error bard represent standard deviation

a

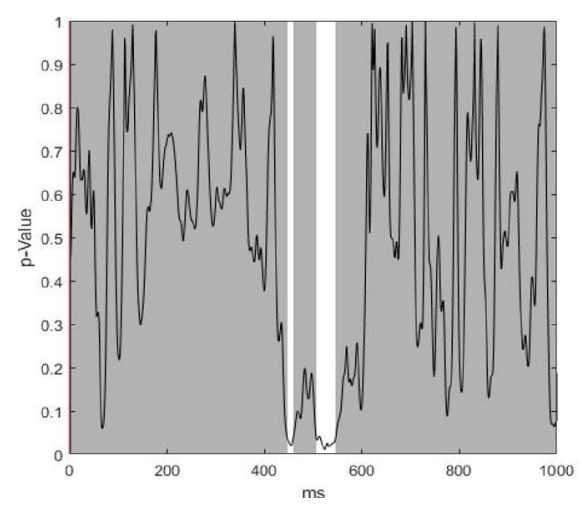

C

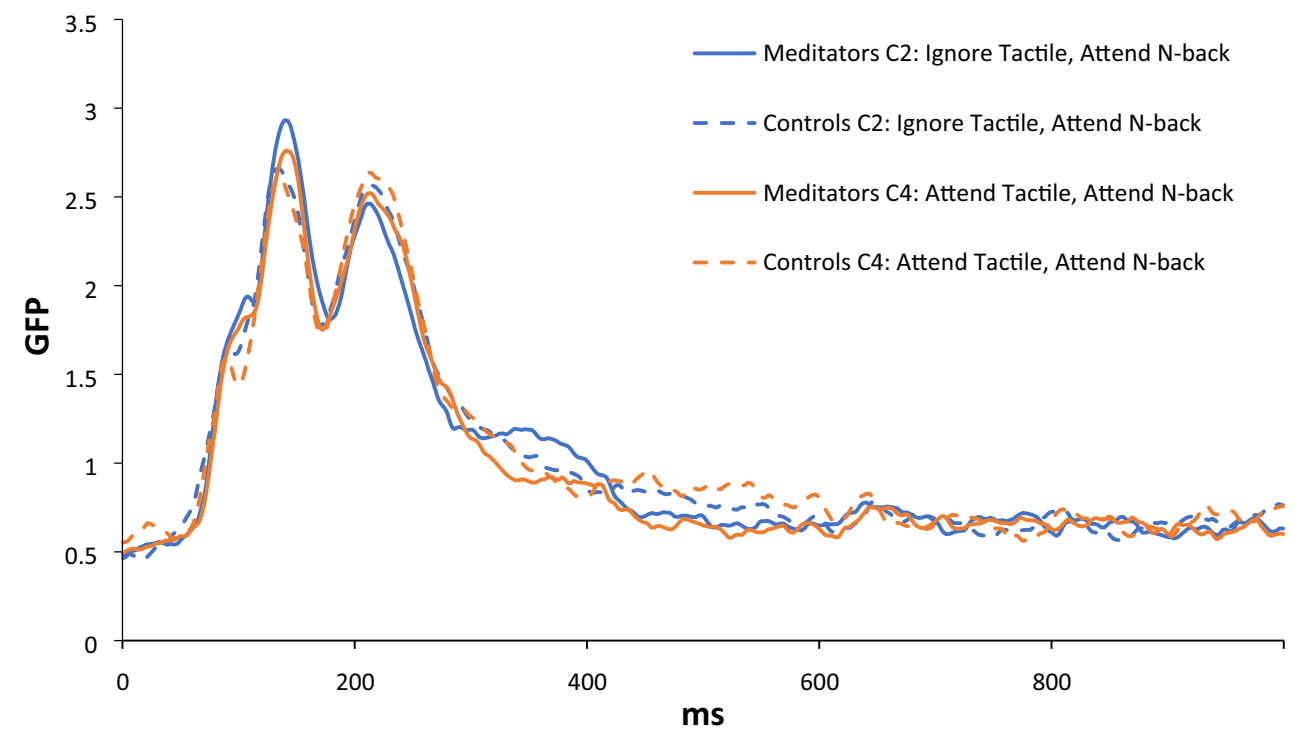

Fig. 4 A) p-graph showing the main effect of group in GFP test across the duration of the epoch. B) p-graph showing the interaction between group and N-back conditions (C2: Tactile, Attend N-back/C4: Attend Tactile, Attend N-back) in the GFP test across the duration of the epoch. C) Average GFP value at each timepoint across the duration of the epoch

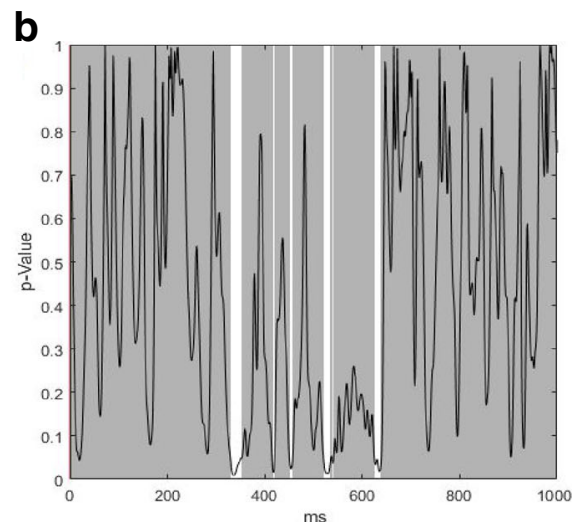

for both groups and N-Back. Note: No significant main effect of Group, nor interaction between Group and N-back conditions in GFP was present in the comparisons across the whole epoch (no significant periods lasted longer than the duration control of $47 \mathrm{~ms}$ ) 
Tactile, Attend N-back conditions). The main effect of group comparison showed a significant difference during two separate time windows, with meditators showing a P300 with a more frontal distribution of positive voltages during both time windows (global count statistics across the whole epoch $p=$ 0.009 FDR $p=0.045)$. A significant main effect of group that survived duration control $(44 \mathrm{~ms})$ for multiple comparisons was found in TANOVA from 299 to $352 \mathrm{~ms}$. This result was significant when activity was averaged across a significant window from 299 to $352 \mathrm{~ms}\left(p=0.005, \eta^{2}=0.060\right)$.

A significant effect of group was also found in TANOVA from 419 to $613 \mathrm{~ms}$, however in light of the TCT results, which showed within group variance among meditators (527-606 ms in C4: Attend Tactile, Attend N-back condition, and from 524-580 ms in C2: Ignore Tactile, Attend N-back condition), the main effect of group from $524 \mathrm{~ms}$ onwards could be due to within group/condition variability. Thus, from 419 to $524 \mathrm{~ms}$ there was a significant main effect between groups ( $p=0.001$, $\eta^{2}=0.076$, global duration control statistic was $\left.44 \mathrm{~ms}\right)$, but after this period, apparent differences between groups may be attributed to variance within the meditator group. Figure 5 depicts topographical differences between groups for the first significant window from 299 to $352 \mathrm{~ms}$, and for the second significant window from 419 to $524 \mathrm{~ms}$. Please refer to Figure S2 in the Supplementary Materials for microstate analysis depicting GFP values and topographies at each timepoint for both groups.

Overall, the differences indicate a P300 with a more central and right frontal-central positivity in the meditation group from 299 to $352 \mathrm{~ms}$, and positive voltages extended further frontally in the meditation group from 419 to $524 \mathrm{~ms}$. Because the P300 typically shows maximal positivity over parietal areas on the scalp and is thought to peak at around $300 \mathrm{~ms}$, the difference in this activity may reflect more frontal regions activated in the meditation group during performance of the function the P300 is associated with - working memory and context updating processes (Kok, 2001; Polich, 2007). No interaction between group and visual WM conditions $(\mathrm{C} 2$ : Ignore Tactile, Attend N-back/C4: Attend Tactile, Attend Nback) was present ( $p>0.05$ for the entire time period, except for a few brief time periods that did not last longer than duration control multiple comparisons, global count statistics across the whole epoch $p=0.106$; FDR $p=0.266$ ).

Bayesian comparison of GFP amplitude limited to the P300 window (300-600 ms) found weak evidence against group differences in P300 GFP amplitude in response to $\mathrm{N}$ back stimuli ( $p=0.33, \eta 2=0.02, \mathrm{BF} 01=1.47)$. However, the Bayesian analysis revealed strong evidence in support of an interaction between group and Visual WM conditions (C2: Ignore Tactile, Attend N-back/C4: Attend Tactile, Attend Nback conditions) $(p=0.003, \eta 2=0.14, \mathrm{BF} 10=13.66)$, with meditators showing a reduced P300 GFP amplitude to the Nback in the dual attend condition compared with single attention condition, whereas controls demonstrated similar P300 GFP amplitude during both conditions (Fig. 6).

\section{Neural Data; Tactile stimulation locked alpha}

\section{Root Mean Square Test}

The RMS test was performed to assess the strength of neural response within the alpha frequency averaged across the 0 to $1000 \mathrm{~ms}$ period for all conditions. No significant main effect of group was present ( $p=0.911$, FDR $p=0.974, \mathrm{BF} 01=2.44$ ), nor interaction between group and Condition 2/Condition 4 (ignore/attend tactile stimuli; $p=0.197$, FDR $p=0.282, \mathrm{BF} 01$ $=1.672$ ). Bayesian analysis of both tests indicate that the results did not distinguish between the null and the alternative hypothesis. A significant interaction was found between group and visual WM N-back task absent/present conditions (C3: Attend Tactile Only/C4: Attend Tactile, Attend N-back) which did not survive FDR multiple comparison control $(p=$ 0.017, FDR $\left.p=0.057, \eta^{2}=0.037, \eta p^{2}=0.062, \mathrm{BF} 10=9.214\right)$. Bayesian analysis found strong evidence in support of the alternative hypothesis, that there was an interaction between the groups.

Post-hoc analyses were performed to explore whether alpha RMS differed during visual (higher WM load) and tactile (lower WM load) conditions. This was conducted by comparing the average of the two N-back conditions were averaged together (C2: Ignore Tactile, Attend N-back/C4: Attend Tactile, Attend N-back) and the average of the two tactile only conditions were averaged together (C1: Ignore Tactile Only and C3: Attend Tactile Only). Post-hoc analysis between groups for averaged N-back conditions $(p=0.3714$, BF01 $=$ 2.654 ) and averaged tactile only conditions ( $p=0.4854$, BF01 $=3.069$ ) were both not significant. However, significant differences were found between the N-back and tactile only conditions within both meditator $\left(p=0.0002, \eta^{2}=0.486\right)$ and control groups $\left(p=0.0002, \eta^{2}=0.277\right)$. It seems that the interaction is driven by the control group showing less difference between the two grouped conditions. RMS test results for the interaction between group and the WM task present/absent conditions are presented in Fig. 7.

\section{TANOVA}

No significant main effect of group was present ( $p=0.154$, FDR $p=0.329$ ), nor main effect of tactile stimuli being ignored/attended ( $p=0.10$ ). Additionally, no significant interaction was found between group and whether tactile stimuli were ignored/attended (C2: Ignore Tactile, Attend N-back/C4: Attend Tactile, Attend N-back; $p=0.974$, FDR $p=0.974)$. A significant interaction was found between group and whether participants were completing the N-back memory task or not (C3: Attend Tactile Only/C4: Attend Tactile, Attend N-back; 


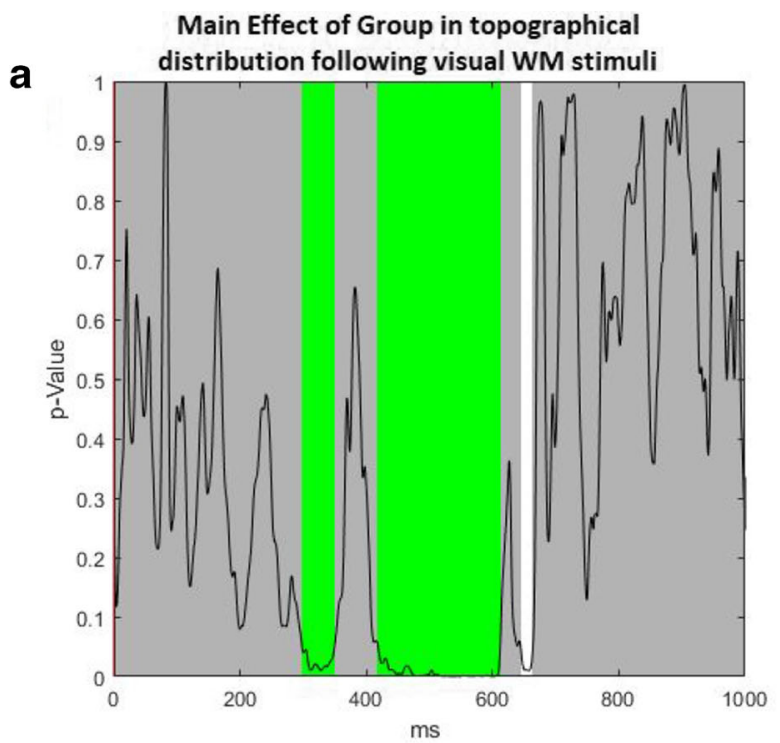

b

\section{9 to $352 \mathrm{~ms}$ time window}
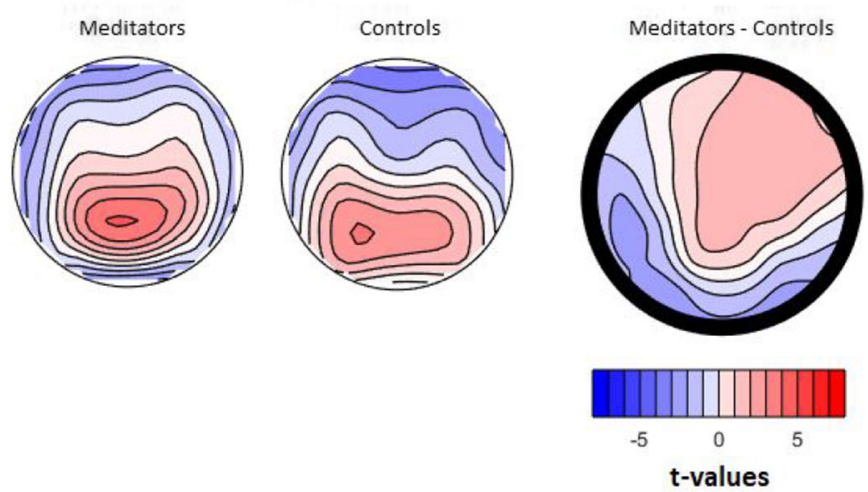

Group effects by N-Back Conditions (Condition 2/Condition 4)

\section{c}

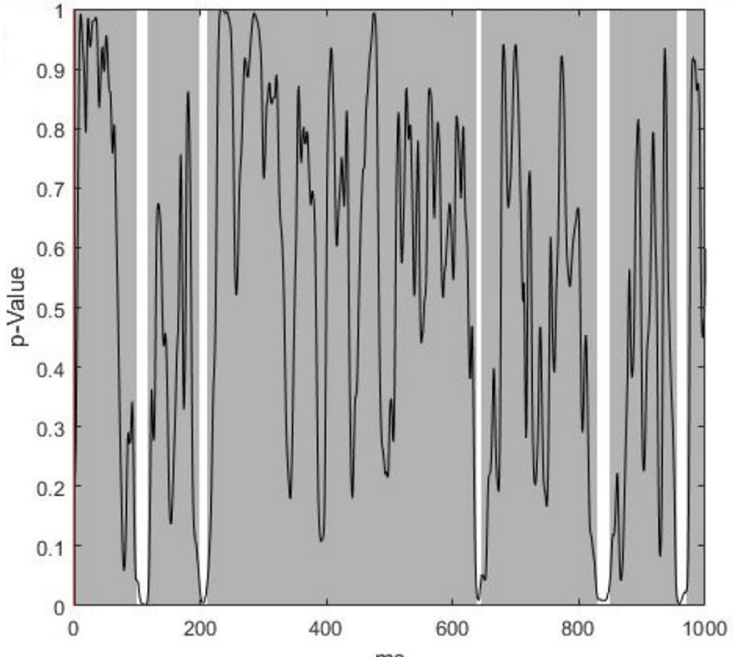

Fig. 5 TANOVA main group effect. A) p-graph showing the significant main effect of group and $p$-values across the duration of the epoch (global count statistics across the whole epoch $p=0.009$, FDR $p=0.045$ ). The probability of the null hypothesis was below 0.05 from 299 to $352 \mathrm{~ms}$ and from 419 to $613 \mathrm{~ms}$. Green bars reflect periods that exceeded the duration control for multiple comparisons across time $(44 \mathrm{~ms})$. This period was a longer duration of significance than $95 \%$ of the 5,000 randomizations. B) Topographic maps for each group and a $t$-map for meditators topography minus control topography during the 299 to $352 \mathrm{~ms}$ time window ( $p=$ 0.005 , averaged across the first significant period, $\eta^{2}=0.060$ ). C) $p$-graph d $\quad 419$ to $524 \mathrm{~ms}$ time window
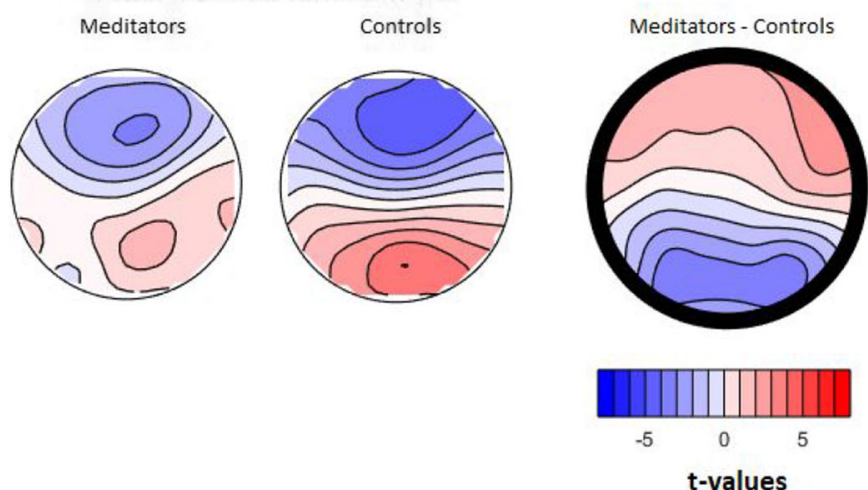

of the interaction between group and ignore/attend N-back condition $(\mathrm{C} 2$ : Ignore Tactile, Attend N-back/C4: Attend Tactile, Attend N-back) across the duration of the epoch. $p>0.05$ for the entire period, except for a few brief time periods that did not last the duration control for multiple comparisons across time (44ms; count statistics across the whole epoch $p=$ $0.11)$. D) Topographic maps for each group and a $t$-map for meditators topography minus control topography during the 419- to 524-ms time window $\left(p=0.001\right.$, averaged across the second significant period, $\eta^{2}=$ $0.076)$

also showed significant differences between conditions ( $p=$ $\left.0.046, \eta^{2}=0.04\right)$, this difference between conditions was not as strong as the difference between conditions found for meditators, indicating that the different strength of the between condition effect between the two groups was perhaps driving the significant interaction.

Post-hoc Exploratory Analysis The RMS test was performed to assess the strength of neural response within the alpha 
口 C2: Ignore Tactile, Attend N-back

- C4: Attend Tactile, Attend N-back

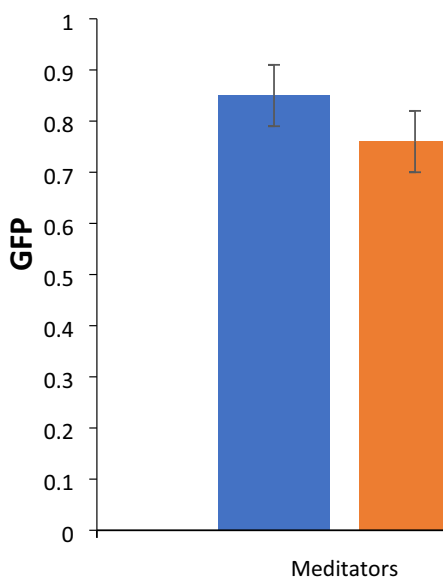

Fig. 6 Averaged GFP values limited to the P300 windows (300-600 ms) for both single and dual attention conditions. There was an interaction between group and condition, with meditators showing a greater difference between conditions than controls $(p=0.003$, $\eta 2=0.14$, $\mathrm{BF} 01=13.66)$. Error bars represent standard error frequency averaged across the 0 to $1000 \mathrm{~ms}$ period for tactile only conditions $(\mathrm{C} 1$ : Ignore Tactile Only and $\mathrm{C} 3$ : Attend Tactile Only). A significant interaction between group and tactile conditions was present $\left(p=0.029, \eta^{2}=0.0791=\eta p^{2}\right.$ $=0.0792, \mathrm{BF} 10=2.098)$. The interaction was driven by the meditation group showing greater alpha modulation during the ignore tactile condition than controls (Results are presented in Fig. 9).

\section{Discussion}

The present study examined whether experienced mindfulness meditators showed differences in neural resource allocation under single and cross-modal task demands. The conditions allowed for examination of differential processing of visual and somatosensory stimuli that required processes including WM, sustained attention, and divided attention. The combina-

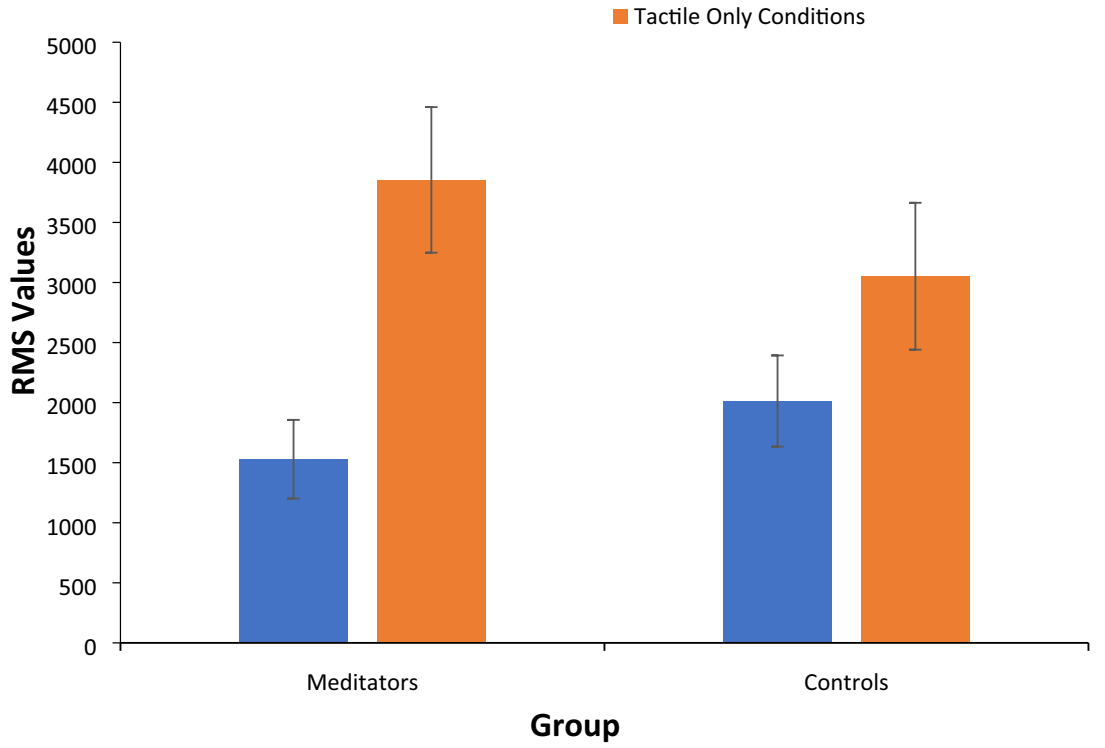

Fig. 7 Averaged alpha activity RMS values across the significant time period 0 to $1,000 \mathrm{~ms}$. Meditators showed a greater difference between conditions than controls $\left(p=0.017\right.$, FDR $p=0.057, \eta^{2}=0.037, \eta^{2}=$ 0.062 ). Significantly larger RMS values in the averaged Tactile Oddball Only conditions than averaged N-back conditions were found for both

meditator $\left(p=0.0002, \eta^{2}=0.486\right)$ and control groups $\left(p=0.0002, \eta^{2}=\right.$ 0.277). Note: $\mathrm{N}-\mathrm{Back}=$ average of conditions with the N-back Task (Conditions 2 and 4), Tactile Only = average of conditions without the N-Back task (Conditions 1 and Condition 3) 
a N-Back
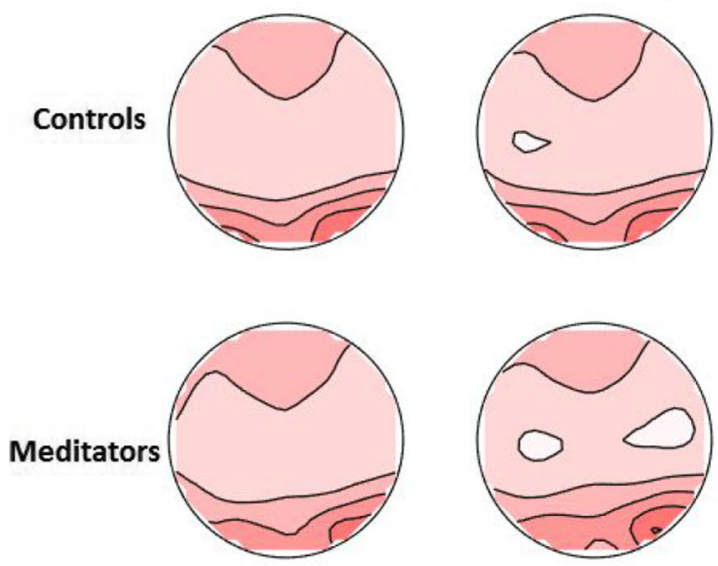

b

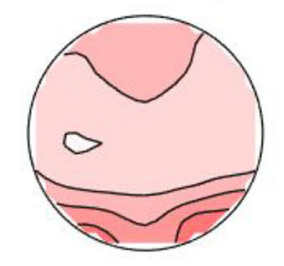

Tactile Only

(

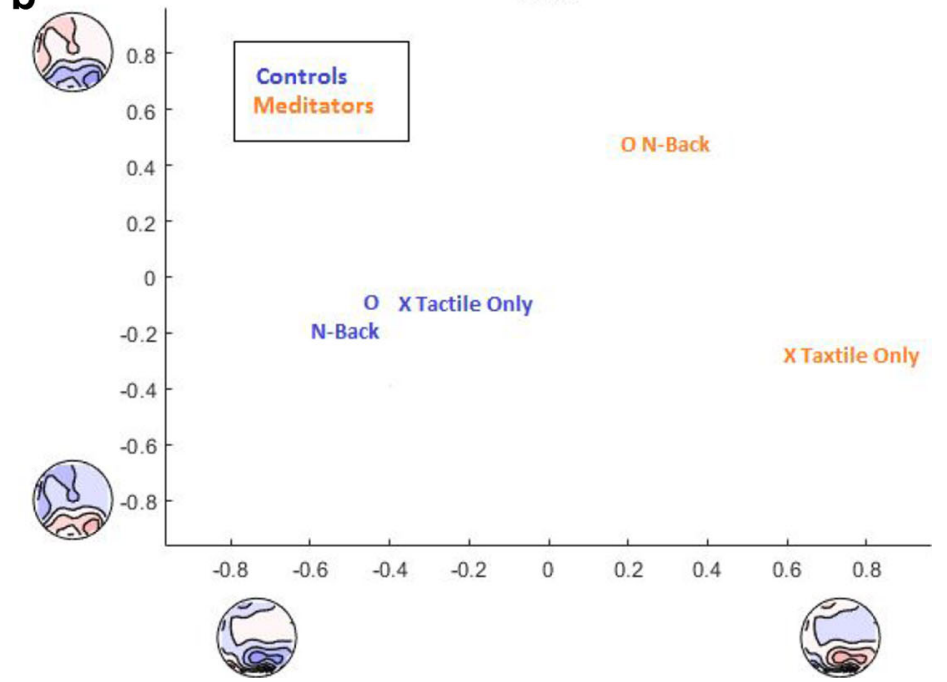

Fig. 8 A) Averaged alpha topographies of Group x N-back/Attend Tactile Only conditions averaged across the 0 to $1,000 \mathrm{~ms}$ window. Meditators showed an altered distribution of alpha activity in the Tactile Only conditions, with more posterior alpha $(p=0.047)$ compared to controls (while groups did not significantly differ in conditions with Nback tasks, $p=0.155$ ). Meditators showed an altered distribution of alpha in the Tactile Only conditions compared to N-back conditions $(p<0.001$, $\left.\eta^{2}=0.19\right)$. Controls also differentiated significantly between conditions,

tion of these processes has not been previously studied in meditators. Meditators showed a P300 with more positive frontal voltages in the early P300, and less negative frontal voltages and less positive parietal voltages in the late P300 when responding to visual stimuli. Meditators also showed greater ability to modulate alpha distribution between low (tactile-only condition) and high (dual task condition) task demands requiring more neural resources. These differences in neural activity were concurrent with improved WM however, this change is smaller than the change observed among meditators $\left(p=0.046, \eta^{2}=0.04\right)$. B - MDS analysis comparing mean alpha topographical maps between groups and Tactile Only/ N-back conditions. The graph indicates that meditators showed more parietal occipital activation during the Attend Tactile Only and more frontal activation during the conditions with N-back. Note: $\mathrm{N}$-Back = average of conditions with the N-back Task, Tactile Only $=$ average of conditions without the NBack task

performance in the meditator group. The results provide evidence of differences in neural activity and resource allocation between meditators compared to demographically-matched controls.

\section{Improved WM}

Existing literature has shown positive effects of mindfulness practice on $\mathrm{WM}$, attention, and cognitive control functions

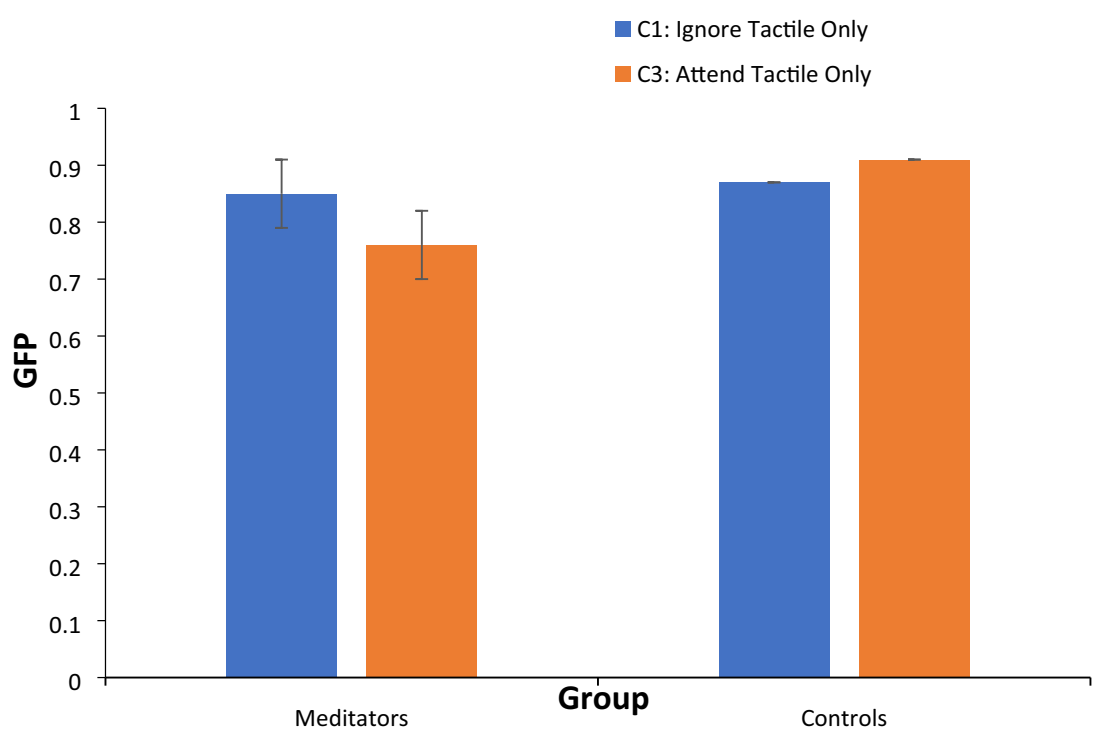

Fig. 9 Averaged alpha activity RMS values across the significant time period 0 to 1,000 ms. Meditators showed a greater difference between conditions than controls $\left(p=0.029, \eta^{2}=0.0791=\eta p^{2}=0.0792\right.$, BF10 $\left.=2.098\right)$ 
(Mrazek, Franklin, Phillips, Baird, \& Schooler, 2013; Quach, Jastrowski Mano, \& Alexander, 2016; Zeidan, Gordon, Merchant, \& Goolkasian, 2010a). The present study adds evidence of improvements in WM functions during divided attention by demonstrating that meditators performing with higher accuracy on the N-back task while simultaneous attending to distractor stimuli. The findings indicate an enhanced ability for meditators to inhibit distraction and distribute neural resources during cognitively demanding task conditions.

\section{More frontally distributed WM ERPs in meditators}

Neural distribution differences between groups during the P300 window are consistent with previous work by Bailey et al. (2018), whom identified a similar pattern of frontal P300 distribution among meditators along with enhancements in attentional control and related behavioural performance during the Go/No-go task. Unique to the present study, source analysis (see Supplementary Materials 1) demonstrated that meditators demonstrated greater neural activity in visuospatial processing regions and less activity in somatic (sense of touch) processing regions in response to visual WM stimuli (Kim et al., 2015; Trés \& Brucki, 2014). The capacity-limited nature of WM functions by modulating neural activity to attend to task-relevant processing regions, and use disengagement mechanisms to guard against interference, enhancing task-relevant information processing (Jha et al., 2019; Shipstead, Harrison, \& Engle, 2016; Sreenivasan \& Jha, 2007). Findings from this study demonstrate that meditators likely attended to the N-back visual stimuli more strongly in both low and high resource demanding conditions, suggesting enhanced ability to orient neural activity to the visual task while simultaneously disengaging from the tactile stimuli.

The enhanced ability among meditators to orient neural activity in favour of task-relevant processing regions is likely to reflect mindfulness practice effects on WM encoding, attention, and cognitive control functions (Kok, 2001; Polich, 2007). In a series of studies on WM and cognitive control, Lavie, de Fockert, and colleagues (De Fockert, Rees, Frith, \& Lavie, 2001; Lavie, 2005; Lavie \& De Fockert, 2005; Lavie, Hirst, De Fockert, \& Viding, 2004) demonstrated that distractor processing increased with greater WM load, and WM load predicted selective attention performance. Using a dual-task study design, participants performed a WM task (memorising a string of numbers) with gradual loading from low to high difficulty (more complex strings of numbers) while simultaneously attending to a visual distractor (e.g. distractor faces). The results showed that increased WM load resulted in greater distractor processing and interference on task performance. The authors theorised that distractor perception is reduced in favour of task relevant processing, however, its ability to guard against interference began to deteriorate with increasing WM demand due to diminishing cognitive control. On this account, the differences in neural activity in meditators in the present study suggest greater capacity in managing high WM loads compared with controls, without compromising distractor reducing functions, suggesting that mindfulness practice may result in more efficient allocation of cognitive control functions during resource demanding tasks.

Results from the present study might indicate differences in the unique neural processes employed by meditators during cognitively demanding tasks. Meditators in the present study demonstrated greater positive neural activity in frontal regions and less positive voltage in parietal regions during the early P300 window, mirroring compensatory neural activity and comparable behavioural performance from studies among the elderly (Friedman, Simpson, \& Hamberger, 1993). However, in the current study this pattern of activity was concurrent with improved behavioural performance in the meditator group. In view of this, our results might suggest that meditators were not simply more proficient at modulating typical WM related neural activity, but that they engaged in a qualitatively different neural process. Greater frontal activity suggests that when performing WM tasks, meditators may have engaged more frontal regions to process and execute task demand and relied less on parietal functions to support related cognitive functions.

\section{P300 Differences Between Groups}

Additionally, an interaction between group and single attention / dual attention conditions was seen in P300 amplitude, with controls showing similar P300 amplitudes toward Nback visual stimuli in both single and dual attention conditions, while mediators demonstrated smaller P300 amplitudes in the dual attention condition compared with the condition where they were only required to attend to the visual WM stimuli. Although this finding was not expected, it is congruent with studies of the attentional-blink in meditators, who show smaller P300 amplitudes toward the first of two attention targets in a rapidly presented sequence of letters, which is thought to enable greater efficiency at allocating attentional resources between targets (in contrast to controls, who show larger P300 amplitudes to the first target, and more commonly miss the second target; Slagter et al., 2007). P300 amplitude and latency are both sensitive to attentional resource availability (Lin, Fisher, \& Moser, 2019; Polich, 2007). The current P300 amplitude results might provide further indication that meditators are able to more effectively split their attention across dual attentional demands, allowing neural resources to be allocated effectively to allow higher performance during greater task demands. It is important to note that although both Frequentist and Bayesian analyses suggested this was a significant result with strong evidence, the analysis limited to the P300 window was exploratory. The planned analysis with 
RAGU indicated the effect was not consistent enough across the epoch to pass global duration controls for multiple comparisons. As such, replication analysing the specific P300 time window is required to confirm the result.

Sustained attention is thought to involve three top-down neural processes: (i) awareness of ongoing cognitive functions, (ii) sustained focus on task-relevant activities, and (iii) suppression of distractions (Clayton, Yeung, \& Cohen Kadosh, 2015). Meditation practice typically involves the focus of attention on present sensations such as the breath entering and leaving the body and refocusing the point of attention each time the mind wanders, training the mind to become more stable (Pregi et al., 2006). This training enhances the ability to supress irrelevant internal stimuli such as ongoing negative ruminations, reflections, and memories, as well as external distractions in the environment (Kerr et al., 2013). Thus, reduced mind wandering and enhanced ability to supress distraction improves present goal-oriented focus and sustained attention. These practice related changes may explain the task-dependent group differences in P300 found in the present study.

\section{Alpha Modulation and Working Memory}

Due to differences in the equipment used to capture EEG data, our results showed a global increase in alpha activity while Kerr et al. (2013) who adopted magnetoencephalography (MEG), which allows for higher accuracy in capturing local data, noted localised changes specific to the somatosensory region. Although EEG captures accurate data globally, it is less sensitive to localised data in specific regions (Lopes da Silva, 2013). Despite this difference, the present study replicated the work by Kerr et al. (2013) with meditators demonstrating greater alpha activity when suppressing/ignoring external (tactile) stimulation than controls. Kerr et al. (2013) theorised that meditators with practice in localised attention to body sensations were likely to have enhanced anticipatory control over somatotopic alpha rhythms, and thus will exhibit greater top-down alpha modulation over sensory filtering brain regions. This theory is consistent with evidence of enhanced attentional regulation and modulation of somatosensory alpha rhythm after short periods of meditative practice, resulting in faster reaction times to cued visual-spatial stimuli (Jha et al., 2007; Kerr et al., 2011).

Meditators also showed more alpha activity in parietaloccipital regions in Tactile Only conditions (non-WM) than conditions with the N-back WM task, indicating greater inhibition of non-relevant visual information processing when the task did not require visual information to be processed. Thus, the current results extend the work by Kerr et al. (2013) to visual processing regions. The results also indicated that both groups showed less alpha when they were concurrently performing the WM task, and that meditators showed more of a decrease between the Tactile Only and N-back (WM) conditions in overall strength of alpha activity. These results suggest that meditators were inhibiting visual processing in the absence of the N-back task and suppressing alpha activity in order to activate visual processing regions during the $\mathrm{N}$ back task when visual processing was required.

\section{Limitations and Future Direction}

Cross-sectional studies face inherent limitations. Selfselection bias in the present study may reflect inherent differences in neurobiological profiles and personality traits that promote uptake or adherence to mindfulness practice (Mascaro, Rilling, Negi, \& Raison, 2013). Additionally, due to the absence of an active control group who are involved in attention intense activities such as learning a language or instrument (Garland \& Howard, 2013), the observed differences in attention regulation between groups may be due to the fact that meditators are experienced in a non-specific form of attention training rather than meditation-specific effects. The most conspicuous potential limitation however, is the difference in years of education between groups, which has been shown to affect cognitive performance and function (Park, Choi, Choi, Kang, \& Lee, 2019). Tests were performed (see Supplementary Materials 2) to assess the effect of this confound by replicating comparisons after groups were matched for years of education, excluding the most educated meditators. The results were consistent with the initial analysis suggesting that education level did not explain the group differences found in the present study.

Potential group differences in motivation were also considered as a potential confounding factor when explaining the results. Previous research in this area has suggested that greater motivation is associated larger P300 amplitudes (CarrilloDe-La-Peña \& Cadaveira, 2000; Franken, van Strien, Bocanegra, \& Huijding, 2011; Hughes, Mathan, \& Yeung, 2013). In our results, no overall group differences were found in P300 amplitudes, and meditators showed reduced P300 amplitudes in the dual attention condition. Furthermore, previous research suggests that increased motivation is associated with faster reaction times (Mir et al., 2011). The control group's average reaction time in each condition was shorter than the meditation group's average reaction time (although not reaching statistical significance), suggesting that the two groups did not differ in their levels of motivation. However, as we did not measure motivation specifically, we cannot eliminate the possibility that a difference in motivation between groups influenced our results.

Finally, mindfulness meditators were recruited based on self-reported practices rather than adherence to an objective, standardised course or set of practices. Efforts were made to screen participants to ensure that their meditation practice contained the defined practice of focused attention, however 
styles of practice still varied with in participants due to the wide variety of mindfulness meditation techniques. While this lack of standardization limits the ability to draw conclusions about a definitive set of practices, it allows the generalizability of our results to many forms of mindfulness meditation. It is also worth commenting that the task required prolonged focus and sustained attention with instructions building on each other gradually increasing in difficulty. Amongst other factors, mindfulness-meditation has been considered helpful for reducing mental fatigue (Kaplan, 2001), with evidence of reduced cognitive fatigue after mindfulness practice (Johansson, Bjuhr, \& Rönnbäck, 2015). Therefore, performance deterioration due to fatigue may have impacted controls more than meditators and may have influenced the results of the study. However, the lack of interaction between group and condition in accuracy across the two N-back conditions suggests fatigue is unlikely to explain our results. Additionally, the exploratory analysis of GFP limited to the P300 windows showed smaller amplitudes of P300 activity in the meditator group during later conditions, a result that could indicate increased fatigue in the meditation group. However, in these conditions the meditation group showed more accurate task performance, suggesting a difference between groups in levels of fatigue is not likely to explain the results

Future research should consider using MEG to capture neural data with higher accuracy for more specifically localised activity. Additionally, further studies are encouraged to determine whether the differential attentional modulation found in the current study is replicable in studies using designs comparing a sham-meditation condition to active meditation training (Johnson, Gur, David, \& Currier, 2013; Noone \& Hogan, 2018), which could eliminate a portion of the limitations set out above and help establish causality.

\section{Summary}

The present research extended existing literature by using EEG markers of attentional resource allocation (P300 and alpha activity; Wong et al., 2018) to demonstrate that meditators were able to differentially allocate neural resources and modulate alpha rhythms to facilitate WM task performance while experiencing a tactile distractor. Attention relevant modulation in somatosensory processing of a tactile stimulation may be particularly significant for understanding mindfulness-specific effects on neural pathways involved in sensory processing, perception, and attenuation (Brown \& Jones, 2010; Gard et al., 2012; Nakamura, Kitagawa, Kawaguchi, \& Tsuji, 1997; Zeidan et al., 2011). Overall, the study's results promote a broader understanding mindfulness meditation by demonstrating unique neural processes employed by meditators during cognitively demanding tasks concurrent with improved WM performance. Further exploration on the subject is needed to identify the mechanism of action underlying this effect.

Open practices statements Data sharing was not approved by the ethics committee as participants did not provide consent to sharing the data with third-party researchers. The experiment was not pre-registered.

\section{Compliance with ethical standards}

Conflict of Interests PBF has received equipment for research from MagVenture A/S, Medtronic Ltd, Cervel Neurotech and Brainsway Ltd and funding for research from Neuronetics and Cervel Neurotech. PBF is on the scientific advisory board for Bionomics Ltd. All other authors have no conflicts to report. PBF is supported by a National Health and Medical Research Council of Australia Practitioner Fellowship 659 (6069070).

\section{References}

Anemüller, J., Sejnowski, T. J., \& Makeig, S. (2003). Complex independent component analysis of frequency-domain electroencephalographic data. Neural Networks, 16(9), 1311-1323.

Baer, R. A., Smith, G. T., Hopkins, J., Krietemeyer, J., \& Toney, L. (2006). Using self-report assessment methods to explore facets of mindfulness. Assessment, 13(1), 27-45.

Bailey, N., Sullivan, C., Rogasch, N., Chung, S., Hoy, K., Van Dam, N., \& Fitzgerald, P. (2018) Mindfulness meditators show altered distributions of early and late neural activity markers of attention in a response inhibition task. BioRxiv, 396259.

Bailey, N. W., Raj, K., Freedman, G., Fitzgibbon, B. M., Rogasch, N. C., Van Dam, N. T., \& Fitzgerald, P. B. (2019). Mindfulness Meditators Do Not Show Differences in Electrophysiological Measures of Error Processing. Mindfulness, 10(7), 1360-1380.

Baldwin, C. L., Roberts, D. M., Barragan, D., Lee, J. D., Lerner, N., \& Higgins, J. S. (2017). Detecting and quantifying mind wandering during simulated driving. Frontiers in Human Neuroscience, 11.

Beck, A. T., \& Clark, D. A. (1997). An information processing model of anxiety: Automatic and strategic processes. Behaviour Research and Therapy, 35(1), 49-58.

Beck, A. T., Steer, R. A., \& Brown, G. K. (1996). Beck Depression Inventory-II. San Antonio, 78(2), 490-498.

Benjamini, Y., \& Hochberg, Y. (1995). Controlling the False Discovery Rate: A Practical and Powerful Approach to Multiple Testing. Journal of the Royal Statistical Society: Series $B$ (Methodological), 57(1), 289-300.

Brown, C. A., \& Jones, A. K. P. (2010). Meditation experience predicts less negative appraisal of pain: Electrophysiological evidence for the involvement of anticipatory neural responses. Pain, 150(3), 428438.

Carrillo-De-La-Peña, M. T., \& Cadaveira, F. (2000). Effet des instructions motivantes sur l'amplitute du P300. Neurophysiologie Clinique, 30(4), 232-239.

Chiesa, A., Calati, R., \& Serretti, A. (2011). Does mindfulness training improve cognitive abilities? A systematic review of neuropsychological findings. Clinical Psychology Review, 31, 449-464.

Clayton, M. S., Yeung, N., \& Cohen Kadosh, R. (2015). The roles of cortical oscillations in sustained attention. Trends in Cognitive Sciences, 19, 188-195.

Compton, R. J., Gearinger, D., \& Wild, H. (2019). The wandering mind oscillates: EEG alpha power is enhanced during moments of mind- 
wandering. Cognitive, Affective and Behavioral Neuroscience, 19(5), 1184-1191.

De Fockert, J. W., Rees, G., Frith, C. D., \& Lavie, N. (2001). The role of working memory in visual selective attention. Science, 291(5509), 1803-1806.

Delorme, A., \& Makeig, S. (2004). EEGLAB: An open source toolbox for analysis of single-trial EEG dynamics including independent component analysis. Journal of Neuroscience Methods, 134(1), 921.

Fox, K. C. R., Dixon, M. L., Nijeboer, S., Girn, M., Floman, J. L., Lifshitz, M., ... Christoff, K. (2016). Functional neuroanatomy of meditation: A review and meta-analysis of 78 functional neuroimaging investigations. Neuroscience and Biobehavioral Reviews, 65, 208-228.

Fox, K. C. R., Nijeboer, S., Dixon, M. L., Floman, J. L., Ellamil, M., Rumak, S. P., ... Christoff, K. (2014). Is meditation associated with altered brain structure? A systematic review and meta-analysis of morphometric neuroimaging in meditation practitioners. Neuroscience and Biobehavioral Reviews, 43, 48-73.

Foxe, J. J., \& Snyder, A. C. (2011). The role of alpha-band brain oscillations as a sensory suppression mechanism during selective attention. Frontiers in Psychology, 2(JUL).

Franken, I. H. A., van Strien, J. W., Bocanegra, B. R., \& Huijding, J. (2011). The $\mathrm{p} 3$ event-related potential as an index ofmotivational relevance a conditioning experiment. Journal of Psychophysiology, 25(1), 32-39.

FRIEDMAN, D., SIMPSON, G., \& HAMBERGER, M. (1993). Agerelated changes in scalp topography to novel and target stimuli. Psychophysiology, 30(4), 383-396.

Gard, T., Hölzel, B. K., Sack, A. T., Hempel, H., Lazar, S. W., Vaitl, D., \& Ott, U. (2012). Pain attenuation through mindfulness is associated with decreased cognitive control and increased sensory processing in the brain. Cerebral Cortex, 22(11), 2692-2702.

Garland, E. L., \& Howard, M. O. (2013). Mindfulness-oriented recovery enhancement reduces pain attentional bias in chronic pain patients. Psychotherapy and Psychosomatics. 82(5), 311-318.

Grieder, M., Crinelli, R. M., Koenig, T., Wahlund, L. O., Dierks, T., \& Wirth, M. (2012). Electrophysiological and behavioral correlates of stable automatic semantic retrieval in aging. Neuropsychologia, $50(1), 160-171$.

Habermann, M., Weusmann, D., Stein, M., \& Koenig, T. (2018). A student's guide to randomization statistics for multichannel eventrelated potentials using Ragu. Frontiers in Neuroscience, $12(\mathrm{JUN})$.

Hughes, G., Mathan, S., \& Yeung, N. (2013). EEG indices of reward motivation and target detectability in a rapid visual detection task. NeuroImage, 64(1), 590-600.

Isbel, B. D., Lagopoulos, J., Hermens, D. F., \& Summers, M. J. (2019). Mental training affects electrophysiological markers of attention resource allocation in healthy older adults. Neuroscience Letters, 698 , 186-191.

Jensen, O., Bonnefond, M., \& VanRullen, R. (2012). An oscillatory mechanism for prioritizing salient unattended stimuli. Trends in Cognitive Sciences, 16, 200-206.

Jensen, O., \& Mazaheri, A. (2010). Shaping functional architecture by oscillatory alpha activity: Gating by inhibition. Frontiers in Human Neuroscience, 4.

Jha, A. P., Denkova, E., Zanesco, A. P., Witkin, J. E., Rooks, J., \& Rogers, S. L. (2019). Does mindfulness training help working memory 'work' better? Current Opinion in Psychology, 28, 273-278.

Jha, A. P., Krompinger, J., \& Baime, M. J. (2007). Mindfulness training modifies subsystems of attention. Cognitive, Affective and Behavioral Neuroscience, 7(2), 109-119.

Johansson, B., Bjuhr, H., \& Rönnbäck, L. (2015). Evaluation of an Advanced Mindfulness Program Following a Mindfulness-Based Stress Reduction Program for Participants Suffering from Mental Fatigue After Acquired Brain Injury. Mindfulness, 6(2), 227-233.
Johnson, S., Gur, R. M., David, Z., \& Currier, E. (2013). One-Session Mindfulness Meditation: A Randomized Controlled Study of Effects on Cognition and Mood. Mindfulness, 6(1), 88-98.

Jones, S. R., Kerr, C. E., Wan, Q., Pritchett, D. L., Hämäläinen, M., \& Moore, C. I. (2010). Cued spatial attention drives functionally relevant modulation of the mu rhythm in primary somatosensory cortex. Journal of Neuroscience, 30(41), 13760-13765.

Kaplan, S. (2001). Meditation, restoration, and the management of mental fatigue. Environment and Behavior, 33(4), 480-506.

Kerr, C. E., Jones, S. R., Wan, Q., Pritchett, D. L., Wasserman, R. H., Wexler, A., ... Moore, C. I. (2011). Effects of mindfulness meditation training on anticipatory alpha modulation in primary somatosensory cortex. Brain Research Bulletin, 85(3-4), 96-103.

Kerr, C. E., Sacchet, M. D., Lazar, S. W., Moore, C. I., \& Jones, S. R. (2013). Title: Mindfulness starts with the body: Somatosensory attention and top-down modulation of cortical alpha rhythms in mindfulness meditation. Frontiers in Human Neuroscience, (JAN).

Kim, J., Müller, K. R., Chung, Y. G., Chung, S. C., Park, J. Y., Bülthoff, H. H., \& Kim, S. P. (2015). Distributed functions of detection and discrimination of vibrotactile stimuli in the hierarchical human somatosensory system. Frontiers in Human Neuroscience, 8(JAN).

Klimesch, W. (2012). Alpha-band oscillations, attention, and controlled access to stored information. Trends in Cognitive Sciences, 16, 606617.

Koenig, T., Kottlow, M., Stein, M., \& Melie-García, L. (2011). Ragu: A free tool for the analysis of EEG and MEG event-related scalp field data using global randomization statistics. Computational Intelligence and Neuroscience, 2011.

Koenig, T., \& Melie-García, L. (2010). A method to determine the presence of averaged event-related fields using randomization tests. Brain Topography, 23(3), 233-242.

Koenig, T., Prichep, L., Lehmann, D., Sosa, P. V., Braeker, E., Kleinlogel, H., ... John, E. R. (2002). Millisecond by millisecond, year by year: Normative EEG microstates and developmental stages. NeuroImage, 16(1), 41-48.

Kok, A. (2001). On the utility of $\mathrm{P} 3$ amplitude as a measure of processing capacity. Psychophysiology, 38(3), 557-577.

Lavie, N. (2005). Distracted and confused?: Selective attention under load. Trends in Cognitive Sciences, 9, 75-82.

Lavie, N., \& De Fockert, J. (2005). The role of working memory in attentional capture. Psychonomic Bulletin and Review, 12(4), 669674.

Lavie, N., Hirst, A., De Fockert, J. W., \& Viding, E. (2004). Load theory of selective attention and cognitive control. Journal of Experimental Psychology: General, 133(3), 339-354.

Lin, Y., Fisher, M. E., \& Moser, J. S. (2019). Clarifying the relationship between mindfulness and executive attention: A combined behavioral and neurophysiological study. Social Cognitive and Affective Neuroscience, 14(2), 205-215.

Linden, D. E. J. (2005). The P300: Where in the brain is it produced and what does it tell us? Neuroscientist, 11, 563-576.

Lippelt, D. P., Hommel, B., \& Colzato, L. S. (2014). Focused attention, open monitoring and loving kindness meditation: Effects on attention, conflict monitoring, and creativity - A review. Frontiers in Psychology, 5.

Lopes da Silva, F. (2013). EEG and MEG: Relevance to neuroscience. Neuron, 80(5), 1112-1128.

Lutz, A., Slagter, H. A., Dunne, J. D., \& Davidson, R. J. (2008). Attention regulation and monitoring in meditation. Trends in Cognitive Sciences, 12, 163-169.

Malinowski, P. (2013). Neural mechanisms of attentional control in mindfulness meditation. Frontiers in Neuroscience, (7 FEB).

Manna, A., Raffone, A., Perrucci, M. G., Nardo, D., Ferretti, A., Tartaro, A., ... Romani, G. L. (2010). Neural correlates of focused attention and cognitive monitoring in meditation. Brain Research Bulletin, $82(1-2), 46-56$. 
Mascaro, J. S., Rilling, J. K., Negi, L. T., \& Raison, C. L. (2013). Preexisting brain function predicts subsequent practice of mindfulness and compassion meditation. NeuroImage, 69, 35-42.

Mir, P., Trender-Gerhard, I., Edwards, M. J., Schneider, S. A., Bhatia, K. P., \& Jahanshahi, M. (2011). Motivation and movement: The effect of monetary incentive on performance speed. Experimental Brain Research, 209(4), 551-559.

Moore, A., Gruber, T., Derose, J., \& Malinowski, P. (2012). Regular, brief mindfulness meditation practice improves electrophysiological markers of attentional control. Frontiers in Human Neuroscience, (JANUARY 2012).

Mrazek, M. D., Franklin, M. S., Phillips, D. T., Baird, B., \& Schooler, J. W. (2013). Mindfulness Training Improves Working Memory Capacity and GRE Performance While Reducing Mind Wandering. Psychological Science, 24(5), 776-781.

Nakamura, H., Kitagawa, H., Kawaguchi, Y., \& Tsuji, H. (1997). Intracortical facilitation and inhibition after transcranial magnetic stimulation in conscious humans. Journal of Physiology, 498(3), 817-823.

Noone, C., \& Hogan, M. J. (2018). A randomised active-controlled trial to examine the effects of an online mindfulness intervention on executive control, critical thinking and key thinking dispositions in a university student sample. BMC Psychology, 6(1).

Palmer, J. A., Makeig, S., Kreutz-Delgado, K., \& Rao, B. D. (2008). Newton method for the ica mixture model. ICASSP, IEEE International Conference on Acoustics, Speech and Signal Processing - Proceedings, 1805-1808.

Park, S., Choi, B., Choi, C., Kang, J. M., \& Lee, J. Y. (2019). Relationship between education, leisure activities, and cognitive functions in older adults. Aging and Mental Health, 23(12), 16511660.

Polich, J. (2007). Updating P300: An integrative theory of P3a and P3b. Clinical Neurophysiology, Vol. 118, pp. 2128-2148.

Pregi, N., Vittori, D., Pérez, G., Leirós, C. P., \& Nesse, A. (2006). Effect of erythropoietin on staurosporine-induced apoptosis and differentiation of SH-SY5Y neuroblastoma cells. Biochimica et Biophysica Acta - Molecular Cell Research, 1763(2), 238-246.

Quach, D., Jastrowski Mano, K. E., \& Alexander, K. (2016). A Randomized Controlled Trial Examining the Effect of Mindfulness Meditation on Working Memory Capacity in Adolescents. Journal of Adolescent Health, 58(5), 489-496.

Rouder, J. N., Morey, R. D., Verhagen, J., Swagman, A. R., \& Wagenmakers, E. J. (2017). Bayesian analysis of factorial designs. Psychological Methods, 22(2), 304-321.

Ruggeri, P., Meziane, H. B., Koenig, T., \& Brandner, C. (2019). A finegrained time course investigation of brain dynamics during conflict monitoring. Scientific Reports.

Sauseng, P., Klimesch, W., Heise, K. F., Gruber, W. R., Holz, E., Karim, A. A., ... Hummel, F. C. (2009). Brain Oscillatory Substrates of Visual Short-Term Memory Capacity. Current Biology, 19(21), 1846-1852.

Scheeringa, R., Petersson, K. M., Oostenveld, R., Norris, D. G., Hagoort, P., \& Bastiaansen, M. C. M. (2009). Trial-by-trial coupling between EEG and BOLD identifies networks related to alpha and theta EEG power increases during working memory maintenance. NeuroImage, 44(3), 1224-1238.

Sheehan, D. V., Lecrubier, Y., Sheehan, K. H., Amorim, P., Janavs, J., Weiller, E., ... Dunbar, G. C. (1998). The Mini-International Neuropsychiatric Interview (M.I.N.I.): The development and validation of a structured diagnostic psychiatric interview for DSM-IV and ICD-10. Journal of Clinical Psychiatry, 59(SUPPL. 20), 22-33.
Shipstead, Z., Harrison, T. L., \& Engle, R. W. (2016). Working Memory Capacity and Fluid Intelligence: Maintenance and Disengagement. Perspectives on Psychological Science, 11(6), 771-799.

Slagter, H. A., Lutz, A., Greischar, L. L., Francis, A. D., Nieuwenhuis, S., Davis, J. M., \& Davidson, R. J. (2007). Mental training affects distribution of limited brain resources. PLoS Biology, 5(6), 12281235 .

Sreenivasan, K. K., \& Jha, A. P. (2007). Selective attention supports working memory maintenance by modulating perceptual processing of distractors. Journal of Cognitive Neuroscience, 19(1), 32-41.

Tabachnick, B.G. and Fidell, L. . (2013). Time-Series Anaylsis. In Using Multivariate Statistics.

Tang, Y. Y., \& Posner, M. I. (2009). Attention training and attention state training. Trends in Cognitive Sciences, Vol. 13, pp. 222-227.

Tanner, D., Morgan-Short, K., \& Luck, S. J. (2015). How inappropriate high-pass filters can produce artifactual effects and incorrect conclusions in ERP studies of language and cognition. Psychophysiology, 52(8), 997-1009.

Thut, G., Nietzel, A., Brandt, S. A., \& Pascual-Leone, A. (2006). $\alpha$-Band electroencephalographic activity over occipital cortex indexes visuospatial attention bias and predicts visual target detection. Journal of Neuroscience, 26(37), 9494-9502.

Trés, E. S., \& Brucki, S. M. D. (2014). Processamento visuoespacial: Uma revisão de conceitos básicos a atuais. Dementia e Neuropsychologia, 8(2), 175-181.

van den Hurk, P. A. M., Giommi, F., Gielen, S. C., Speckens, A. E. M., \& Barendregt, H. P. (2010). Greater efficiency in attentional processing related to mindfulness meditation. Quarterly Journal of Experimental Psychology, 63(6), 1168-1180.

van Leeuwen, S., Singer, W., \& Melloni, L. (2012). Meditation increases the depth of information processing and improves the allocation of attention in space. Frontiers in Human Neuroscience, (MAY 2012).

Walach, H., Buchheld, N., Buttenmüller, V., Kleinknecht, N., \& Schmidt, S. (2006). Measuring mindfulness-the Freiburg Mindfulness Inventory (FMI). Personality and Individual Differences, 40(8), $1543-1555$.

Walhovd, K. B., \& Fjell, A. M. (2003). The relationship between P3 and neuropsychological function in an adult life span sample. Biological Psychology, 62(1), 65-87.

Wickens, T. D. (2010). Elementary signal detection theory. In Elementary Signal Detection Theory.

Wong, K. F., Teng, J., Chee, M. W. L., Doshi, K., \& Lim, J. (2018). Positive effects of mindfulness-based training on energy maintenance and the EEG correlates of sustained attention in a cohort of nurses. Frontiers in Human Neuroscience, 12.

Zeidan, F., Gordon, N. S., Merchant, J., \& Goolkasian, P. (2010a). The Effects of Brief Mindfulness Meditation Training on Experimentally Induced Pain. Journal of Pain.

Zeidan, F., Johnson, S. K., Diamond, B. J., David, Z., \& Goolkasian, P. (2010b). Mindfulness meditation improves cognition: Evidence of brief mental training. Consciousness and Cognition, 19(2), 597605.

Zeidan, F., Martucci, K. T., Kraft, R. A., Gordon, N. S., Mchaffie, J. G., \& Coghill, R. C. (2011). Brain mechanisms supporting the modulation of pain by mindfulness meditation. Journal of Neuroscience, $31(14), 5540-5548$.

Publisher's note Springer Nature remains neutral with regard to jurisdictional claims in published maps and institutional affiliations. 